\title{
The Prevalence and Incidence of Dementia Due to Alzheimer's Disease: a Systematic Review and Meta-Analysis
}

\author{
Kirsten M. Fiest, Jodie I. Roberts, Colleen J. Maxwell, David B. Hogan, \\ Eric E. Smith, Alexandra Frolkis, Adrienne Cohen, Andrew Kirk, Dawn Pearson, \\ Tamara Pringsheim, Andres Venegas-Torres, Nathalie Jetté
}

\begin{abstract}
Background: Updated information on the epidemiology of dementia due to Alzheimer's disease (AD) is needed to ensure that adequate resources are available to meet current and future healthcare needs. We conducted a systematic review and meta-analysis of the incidence and prevalence of AD. Methods: The MEDLINE and EMBASE databases were searched from 1985 to 2012 , as well as the reference lists of selected articles. Included articles had to provide an original population-based estimate for the incidence and/or prevalence of AD. Two individuals independently performed abstract and full-text reviews, data extraction and quality assessments. Random-effects models were employed to generate pooled estimates stratified by age, sex, diagnostic criteria, location (i.e., continent) and time (i.e., when the study was done). Results: Of 16,066 abstracts screened, 707 articles were selected for full-text review. A total of 119 studies met the inclusion criteria. In community settings, the overall point prevalence of dementia due to AD among individuals 60 + was 40.2 per 1000 persons $\left(\mathrm{CI}_{95 \%}\right.$ : 29.1-55.6), and pooled annual period prevalence was 30.4 per 1000 persons $\left(\mathrm{CI}_{95 \%}\right.$ : $\left.15.6-59.1\right)$. In community settings, the overall pooled annual incidence proportion of dementia due to AD among individuals $60+$ was 34.1 per 1000 persons $\left(\mathrm{CI}_{95 \%}\right.$ : 16.4-70.9), and the incidence rate was 15.8 per 1000 person-years $\left(\mathrm{CI}_{95 \%}\right.$ : 12.9-19.4). Estimates varied significantly with age, diagnostic criteria used and location (i.e., continent). Conclusions: The burden of AD dementia is substantial. Significant gaps in our understanding of its epidemiology were identified, even in a high-income country such as Canada. Future studies should assess the impact of using such newer clinical diagnostic criteria for AD dementia such as those of the National Institute on Aging-Alzheimer's Association and/or incorporate validated biomarkers to confirm the presence of Alzheimer pathology to produce more precise estimates of the global burden of AD.
\end{abstract}

RÉSUMÉ: Prévalence et incidence de la démence due à la maladie d'Alzheimer : revue systématique et méta-analyse. Contexte: Nous avons besoin d'informations sur l'épidémiologie de la démence due à la maladie d'Alzheimer (MA) afin de nous assurer que des ressources adéquates sont disponibles pour satisfaire les besoins actuels et futurs de la population en soins de santé. Nous avons effectué une revue systématique et une méta-analyse de l'incidence et de la prévalence de la MA. Méthodologie: Nous avons effectué une recherche dans les bases de données MEDLINE et EMBASE de 1985 à 2012 ainsi que dans la liste de références d'articles retenus. Les articles retenus devaient fournir des estimations de l'incidence et/ou de la prévalence populationnelle de la MA. Deux évaluateurs ont revu indépendamment les résumés et le texte intégral ainsi que l'extraction des données des publications et en ont évalué la qualité. Nous avons utilisé des modèles à effets aléatoires pour générer des estimations regroupées stratifiées par âge, sexe, critères diagnostiques, lieu (continent) et temps (moment où l'étude a été réalisée). Résultats: Parmi les 16066 résumés examinés, 707 articles ont été retenus pour une revue du texte intégral. En tout, 119 études rencontraient les critères d'inclusion. Dans la communauté, la prévalence ponctuelle globale de la démence due à la MA chez les individus de 60 ans et plus était de 40,2 par 1000 (IC à 95\%: 29,1 à 55,6) et la prévalence annuelle pour les données regroupées était de 30,4 par 1000 (IC à 95\%: 15,6 à 59,1). Dans la communauté, la proportion d'incidence annuelle globale regroupée de la démence due à la MA chez les individus de 60 ans et plus était de 34,1 par 1000 (IC à 95\%: 16,4 à 70,9) et le taux d'incidence était de 15,8 par 1000 personnes-années (IC à 95\%: 12,9 à 19,4). Les estimations variaient significativement selon l'âge, les critères diagnostiques utilisés et le lieu (continent). Conclusions: Le fardeau de la démence dû à la MA est considérable. Nous avons identifié des lacunes importantes dans notre compréhension de son épidémiologie, même dans un pays à revenu élevé comme le Canada. Des études ultérieures devraient évaluer l'impact de l'utilisation de critères diagnostiques plus récents pour identifier la démence due à la

From the Department of Community Health Sciences, University of Calgary, Calgary, Alberta, Canada (KMF, JIR, CJM, EES, AF, TP, NJ); Department of Clinical Neurosciences,

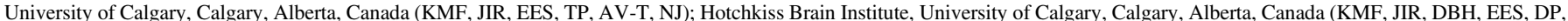

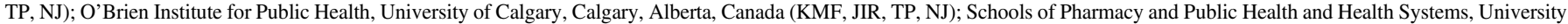

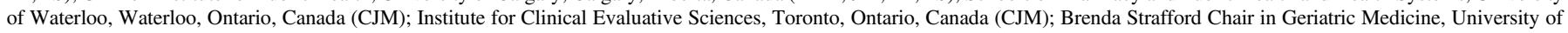

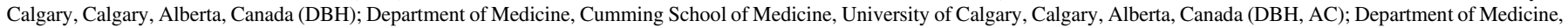
University of Saskatchewan, Saskatoon, Saskatchewan, Canada (AK).

Received OCtOBer 14, 2015. Final Revisions SubmitTed February 22, 2016.

Correspondence to: Nathalie Jetté, Foothills Medical Center, Department of Clinical Neurosciences, 1403-29th Street NW, Calgary, Alberta T2N 4N1, Canada. Email: Nathalie. jette@albertahealthservices.ca. 
MA tels ceux du National Institute on Aging-Alzheimer's Association et/ou incorporer des biomarqueurs validés pour confirmer la présence de la pathologie de la MA et fournir des estimations plus précises de son fardeau global.

Keywords: Alzheimer's, dementia, meta-analysis, systematic review

doi:10.1017/cjn.2016.36

Can J Neurol Sci. 2016; 43: S51-S82

\section{INTRODUCTION}

Alzheimer's disease (AD) is a progressive neurodegenerative disorder leading to cognitive impairment, neuropsychiatric symptoms, disability, dependency, caregiver burden, substantial healthcare expenditures and premature death. ${ }^{1-3}$ Up to $70 \%$ of the dementias occurring in older adults are attributed in whole or in part to AD. ${ }^{4}$ Though described more than a century ago, ${ }^{5}$ treatment options remain limited. Available pharmacotherapies provide modest symptomatic benefits ${ }^{6}$ of debatable cost-effectiveness. ${ }^{7}$

Updated information on the epidemiology of dementia due to $\mathrm{AD}$ is needed if we are to ensure that adequate resources are mobilized to deal with the needs of those with this condition and their families. Such studies can also inform prevention strategies and approaches to management. Systematic reviews on the epidemiology of dementia generally do not deal with specific causes such as $\mathrm{AD}$, but rather provide estimates of overall dementia. ${ }^{8,9}$ The last systematic review of the global incidence of dementia specifically due to $\mathrm{AD}$ was published in 2008. ${ }^{10}$ While the age-specific incidence rate of $\mathrm{AD}$ dementia doubles approximately every 5.5 years in older populations ${ }^{11}$ and several studies have produced estimates stratified by sex and geographic region, ${ }^{10,12-14}$ an unexplored issue is the heterogeneity produced by differing diagnostic criteria and study setting. In a majority of the more recent reviews, either a systematic methodology was not utilized ${ }^{10,12,15}$ or it was uncertain whether one was. ${ }^{14,16,17}$

In this report, we present an updated systematic review and meta-analysis of population-based studies of the incidence and prevalence of dementia due to $\mathrm{AD}$. We also examine the extent and causes of heterogeneity in these estimates.

\section{MethodS}

This is one in a series of systematic reviews on the prevalence and incidence of priority neurological conditions as part of the National Population Health Study of Neurological Conditions. ${ }^{18}$

\section{Search Strategy}

The systematic review and meta-analysis were conducted according to a predetermined protocol based on the PRISMA statement for systematic reviews and meta-analyses. ${ }^{19}$ The search strategy (Appendix A) was developed by the study authors (who have expertise in dementia and/or disease epidemiology) in consultation with a research librarian with systematic review expertise. The primary search was conducted in the MEDLINE and EMBASE databases in February of 2011 and updated in July of 2012. References were exported and managed using EndNote $\mathrm{X} 5 .^{20}$ International studies published before the year 2000 and Canadian studies published prior to 1985 were excluded because of the availability of prior meta-analyses summarizing earlier work. The earlier date for Canadian studies was to ensure that all relevant national work was included, as this was part of a nationally funded study examining the burden of neurological conditions in Canada. The review was restricted to articles published in English or French. The reference lists of included articles were manually searched for additional articles.

\section{Study Selection}

Two reviewers independently examined the titles and abstracts of all retrieved references in order to identify papers likely reporting original population-based data on the prevalence and/or incidence of $\mathrm{AD}$ dementia. Two reviewers also independently examined the fulltext papers identified during the first phase. To be included in the systematic review, reviewed papers had to: (1) report original research; (2) be population-based; and (3) provide an incidence and/ or prevalence estimate of dementia due to AD. Disagreements about the inclusion of articles were resolved by consensus or involvement of a third author if necessary.

\section{Data Extraction and Study Quality}

Two reviewers extracted data from included articles using a standard data collection form. Any disagreement was resolved by consensus. When multiple articles reported data on the same study population, the most accurate and comprehensive data as determined by the reviewers were used. In cases where the studies reported on different data collection years or subgroups (e.g., by sex and/or age), all data were included. The demographic data recorded included age, sex, setting (community-only, both community and institution, institution-only) and study location (i.e., Africa, Asia, Australia, Europe, North America, South America). The approach to ascertain cases was noted, as were sources of data and definitions/diagnostic criteria used. Incidence and prevalence estimates of AD dementia from each study were recorded, along with any stratification by age, sex or year of data collection. The quality of the included studies was evaluated using an assessment tool ${ }^{21,22}$ (Appendix B), with each study given a quality score that ranged from 0 to 8 (higher scores indicating a higher-quality assessment).

\section{Data Synthesis and Analysis}

The significance of age, sex, diagnostic criteria, location (i.e., continent) and time (i.e., when the study was conducted) on incidence and prevalence estimates was assessed using metaregression. Age was examined using the youngest-aged person in the study as a continuous factor of potential heterogeneity (note that few studies provided data on mean or median age). Sex, diagnostic criteria and geographic location were treated as categorical variables. Changes over time were examined using the study start, midpoint and end-years as potential sources of heterogeneity. All pooled estimates were restricted to studies reporting on older individuals (i.e., aged $60+, 65+, 70+$ ) to mitigate the potential confounding effects of age. All period estimates were converted to annual estimates (e.g., period prevalence represents the annual period prevalence). Studies were also stratified by the location of participants (i.e., communityonly, community and institution, institution-only) to minimize 
confounding by disease severity. Studies were included in the meta-analysis if they reported the estimate with $95 \%$ confidence intervals $\left(\mathrm{CI}_{95 \%}\right)$, the number of $\mathrm{AD}$ cases along with overall sample size, or the information with which to calculate an estimate. Additionally, subgroup meta-analysis was only performed if more than one study was available for each subgroup (e.g., a region could have been omitted from this analysis if only one study was available in a region; however, if more than one study was included in the other regions, these data were then analyzed).

To compare study quality characteristics across groups (i.e., continent), ANOVA testing was utilized to determine differences. To assess for significant between-study heterogeneity, the Cochrane $Q$ statistic was calculated and $I^{2}$ was employed to quantify the magnitude. All the pooled estimates and 95\% confidence intervals were calculated using random-effects models. Publication bias was investigated visually using funnel plots and statistically using Begg's, ${ }^{23}$ Egger's ${ }^{24}$ and the trim-andfill tests. The trim-and-fill method identifies funnel plot asymmetry by imputing the effect estimates of potentially missing studies and assessing the influence of these studies on the pooled estimate. For all tests, a value of $p$ less than 0.05 was deemed to be significant. All statistical analyses were carried out in $R$ version 2.14 . $^{25}$ The meta package was used to produce the pooled estimates, forest plots and publication bias assessments. ${ }^{26}$ The metafor package was used to conduct the meta-regression using restricted maximum-likelihood estimation. ${ }^{27}$

\section{RESUlts \\ Identification and Description of Studies}

The search strategy yielded a total of 16,066 citations, including duplicates (8743 from MEDLINE, 7323 from EMBASE) (Figure 1). After screening titles and abstracts, 707 articles were selected for full-text review. Of them, 547 were excluded (230 international studies were published before 2000 , 164 did not report incidence or prevalence of dementia, 114 were not population-based, 39 did not report original data). The updating of the search and hand searching the references led to an additional 4 and 12 articles, respectively. Among the 176 eligible papers meeting the inclusion criteria, 57 were excluded, as they did not report on the incidence or prevalence of $\mathrm{AD}$ dementia. A total of 119 papers reported on AD dementia.

The characteristics of the 119 included studies are shown in Tables 1-3. Seventy-five reported on prevalence, 43 on incidence and 1 on both. Forty-four studies provided data from Europe,

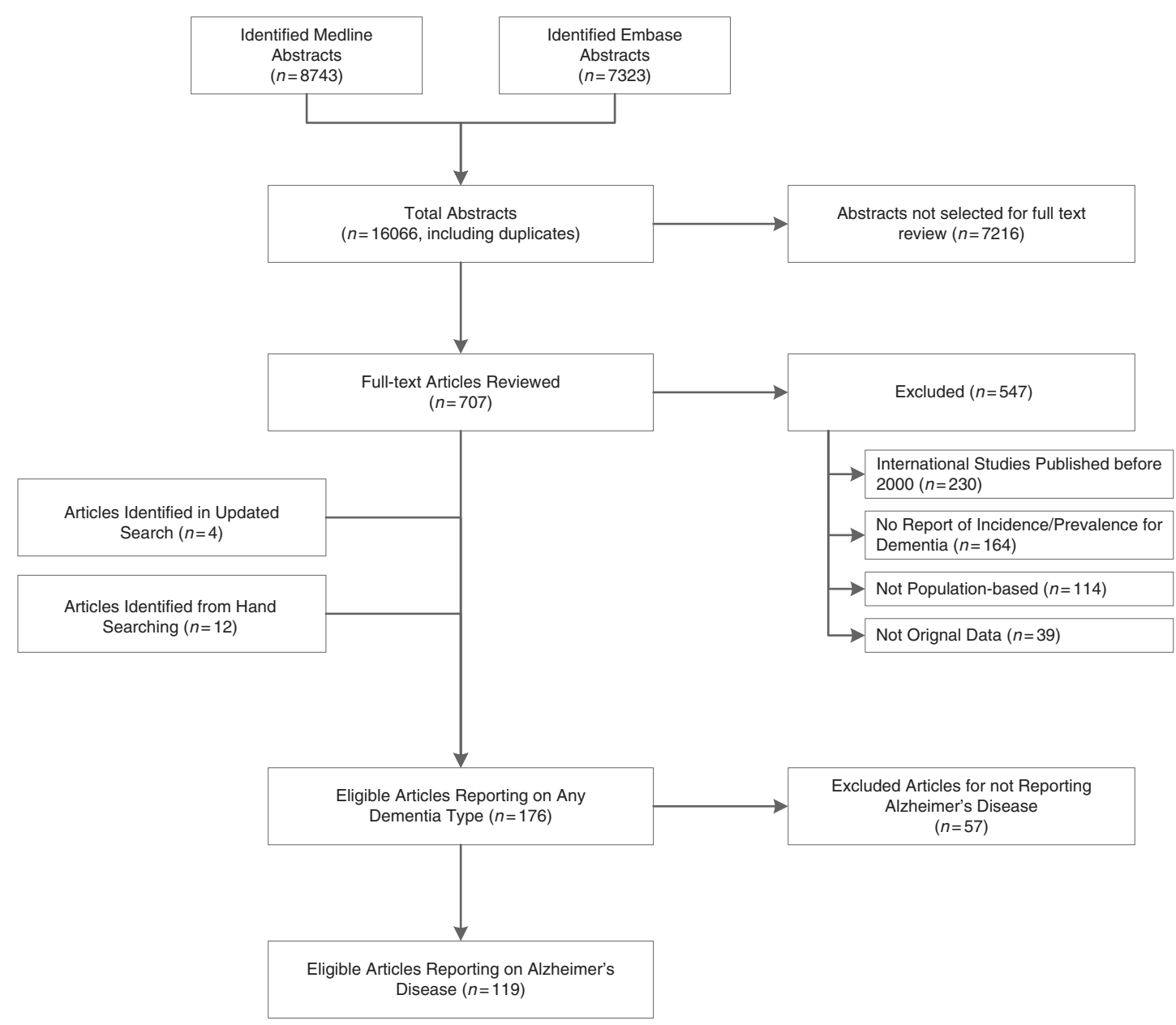

Figure 1: Study flow diagram. 
Table 1: Studies Reporting on the Prevalence of Alzheimer's Disease

\begin{tabular}{|c|c|c|c|c|c|c|c|}
\hline Author, Date & Country and Region & $\begin{array}{l}\text { Age Range } \\
\text { Studied }\end{array}$ & Data Source & $\begin{array}{l}\text { Diagnosis } \\
\text { Established by }\end{array}$ & Diagnostic Criteria & $\begin{array}{l}\text { Years of Data } \\
\text { Collection }\end{array}$ & Groups Studied \\
\hline \multicolumn{8}{|l|}{ Community Only } \\
\hline Anttila (2004) & $\begin{array}{l}\text { FINLAND } \\
\text { Kupio and Joensuu }\end{array}$ & $65-79$ & Cannot determine & Health professional & NINCDS-ADRDA & 1998 & Overall \\
\hline Banerjee (2008) & $\begin{array}{l}\text { INDIA } \\
\text { Kolkata }\end{array}$ & $50+$ & Door-to-Door survey & Health professional & NINCDS-ADRDA & $2002-2003$ & Overall \\
\hline Bermejo-Pareja (2009) & $\begin{array}{l}\text { SPAIN } \\
\text { Las Margaritas, Lista, Arevalo }\end{array}$ & $65+$ & $\begin{array}{l}\text { Telephone Survey } \\
\text { Mailed survey }\end{array}$ & $\begin{array}{l}\text { Health professional } \\
\text { Administrative data } \\
\text { codes } \\
\text { Medical chart review }\end{array}$ & NINCDS-ADRDA & 1994-1998 & Overall \\
\hline Borjesson-Hanson (2004) & $\begin{array}{l}\text { SWEDEN } \\
\text { Goteborg }\end{array}$ & 95 & Census & $\begin{array}{l}\text { Health professional } \\
\text { Medical chart review }\end{array}$ & NINCDS-ADRDA & 1996-1998 & $\begin{array}{l}\text { Male Overall } \\
\text { Female Overall } \\
\text { Overall }\end{array}$ \\
\hline Bottino (2008) & $\begin{array}{l}\text { BRAZIL } \\
\text { Sao Paulo }\end{array}$ & $60+$ & Door-to-Door survey & $\begin{array}{l}\text { Health professional } \\
\text { Imaging test }\end{array}$ & DSM-IV & $2002-2003$ & Overall \\
\hline Bowirrat (2001) & $\begin{array}{l}\text { ISRAEL } \\
\text { Wadi Ara }\end{array}$ & $60+$ & Door to Door Survey & Health professional & DSM-IV & 1995 & Overall \\
\hline Bowirrat (2002) & $\begin{array}{l}\text { ISRAEL } \\
\text { Wadi Ara }\end{array}$ & $60+$ & Door to Door Survey & Health professional & DSM-IV & 1995 & $\begin{array}{l}\text { Male Overall } \\
\text { Female Overall } \\
\text { Overall }\end{array}$ \\
\hline Bowirrat (2002) & $\begin{array}{l}\text { ISRAEL } \\
\text { Wadi Ara }\end{array}$ & $60+$ & Door to Door Survey & Health professional & DSM-IV & 1995 & $\begin{array}{l}\text { Male Overall } \\
\text { Female Overall } \\
\text { Overall }\end{array}$ \\
\hline $\begin{array}{l}\text { Canadian Study of Health } \\
\text { and Aging Working } \\
\text { Group (1994) }\end{array}$ & CANADA & $65+$ & $\begin{array}{l}\text { Administrative } \\
\text { Database }\end{array}$ & Health professional & NINCDS-ADRDA & 1991 & $\begin{array}{l}\text { Male 85+ } \\
\text { Female 85+ } \\
85+ \\
\text { Male 65-74 } \\
\text { Male 75-84 } \\
\text { Male 85+ } \\
\text { Male Overall } \\
\text { Female 65-74 } \\
\text { Female 75-84 } \\
\text { Female 85+ } \\
\text { Female Overall } \\
\text { 65-74 } \\
75-84 \\
85+ \\
\text { Overall }\end{array}$ \\
\hline Dahl (2007) & SWEDEN & $70-81$ & Registry & $\begin{array}{l}\text { Health professional } \\
\text { Administrative data } \\
\text { codes }\end{array}$ & DSM-IV & $2001-2005$ & Overall \\
\hline Das (2006) & $\begin{array}{l}\text { INDIA } \\
\text { Kolkata } \\
\end{array}$ & $50+$ & Door-to-Door survey & Health professional & NINCDS-ADRDA & 2003-2004 & Overall \\
\hline Das (2008) & $\begin{array}{l}\text { INDIA } \\
\text { Kolkata }\end{array}$ & $60+$ & Door-to-Door survey & Health professional & NINCDS-ADRDA & $2003-2004$ & Overall \\
\hline de Jesus Llibre (2009) & CUBA & $65+$ & $\begin{array}{l}\text { Door-to-Door survey } \\
\text { Registry }\end{array}$ & $\begin{array}{l}\text { Health professional } \\
\text { Imaging test } \\
\text { Other }\end{array}$ & NINCDS-ADRDA & 2003 & Overall \\
\hline
\end{tabular}


Table 1. (Continued)

\begin{tabular}{|c|c|c|c|c|c|c|c|}
\hline Author, Date & Country and Region & $\begin{array}{c}\text { Age Range } \\
\text { Studied }\end{array}$ & Data Source & $\begin{array}{l}\text { Diagnosis } \\
\text { Established by }\end{array}$ & Diagnostic Criteria & $\begin{array}{l}\text { Years of Data } \\
\text { Collection }\end{array}$ & Groups Studied \\
\hline Jhoo (2008) & $\begin{array}{l}\text { KOREA } \\
\text { Seongnam }\end{array}$ & $65+$ & $\begin{array}{l}\text { Mailed survey } \\
\text { Telephone survey }\end{array}$ & $\begin{array}{l}\text { Health professional } \\
\text { Imaging test } \\
\text { Other }\end{array}$ & NINCDS-ADRDA & $2005-2006$ & $\begin{array}{l}65-69 \\
70-74 \\
75-79 \\
80+ \\
\text { Male Overall } \\
\text { Female Overall } \\
\text { Overall }\end{array}$ \\
\hline Kivipelto (2001) & $\begin{array}{l}\text { FINLAND } \\
\text { Kupio and Joensuu }\end{array}$ & $65-79$ & Census & $\begin{array}{l}\text { Health professional } \\
\text { Self-report }\end{array}$ & NINCDS-ADRDA & 1998 & Overall \\
\hline Kivipelto (2002) & $\begin{array}{l}\text { FINLAND } \\
\text { Kupio and Joensuu }\end{array}$ & $65-79$ & Census & Health professional & NINCDS-ADRDA & 1998 & Overall \\
\hline Lee (2002) & KOREA & $65+$ & Door-to-Door survey & $\begin{array}{l}\text { Health professional } \\
\text { Medical chart review } \\
\text { Imaging test }\end{array}$ & NINCDS-ADRDA & $1999-2000$ & $\begin{array}{l}65-69 \\
70-74 \\
75-79 \\
80-84 \\
85+ \\
\text { Overall } \\
\end{array}$ \\
\hline $\operatorname{Li}(2007)$ & $\begin{array}{l}\text { USA } \\
\text { Chicago }\end{array}$ & $65+$ & $\begin{array}{l}\text { Health Maintenance } \\
\text { Organization }\end{array}$ & $\begin{array}{l}\text { Health professional } \\
\text { Imaging test }\end{array}$ & NINCDS-ADRDA & 1994-1996 & Overall \\
\hline Lopez (2003) & $\begin{array}{l}\text { USA } \\
\text { Pittsburgh, Sacramento, Winston- } \\
\quad \text { Salem, } \\
\text { Hagerstown }\end{array}$ & $65+$ & $\begin{array}{l}\text { Administrative } \\
\text { databases } \\
\text { Other }\end{array}$ & Health professional & NINCDS-ADRDA & 1998-1999 & Overall \\
\hline Maneno (2006) & USA & $60+$ & $\begin{array}{l}\text { Administrative } \\
\text { databases }\end{array}$ & $\begin{array}{l}\text { Administrative data } \\
\text { codes }\end{array}$ & ICD-9 & $2000-2002$ & Overall \\
\hline Mathuranath (2010) & $\begin{array}{l}\text { INDIA } \\
\text { Kerala }\end{array}$ & $55+$ & Door-to-Door survey & Health professional & $\begin{array}{l}\text { NINCDS-ADRDA } \\
\text { DSM-IV }\end{array}$ & 2001 & $\begin{array}{l}55-59 \\
60-64 \\
65-69 \\
70-74 \\
75-79 \\
80-84 \\
85+ \\
65+ \\
\text { Overall } \\
\text { Male 55-59 } \\
\text { Male 60-64 } \\
\text { Male 65-69 } \\
\text { Male 70-74 } \\
\text { Male 75-79 } \\
\text { Male 80-84 } \\
\text { Male 85+ } \\
\text { Male 65+ } \\
\text { Male Overall } \\
\text { Female 55-59 } \\
\text { Female 60-64 } \\
\text { Female 65-69 } \\
\text { Female 70-74 } \\
\text { Female 75-79 } \\
\text { Female 80-84 } \\
\text { Female 85+ } \\
\text { Female 65+ } \\
\text { Female Overall }\end{array}$ \\
\hline
\end{tabular}




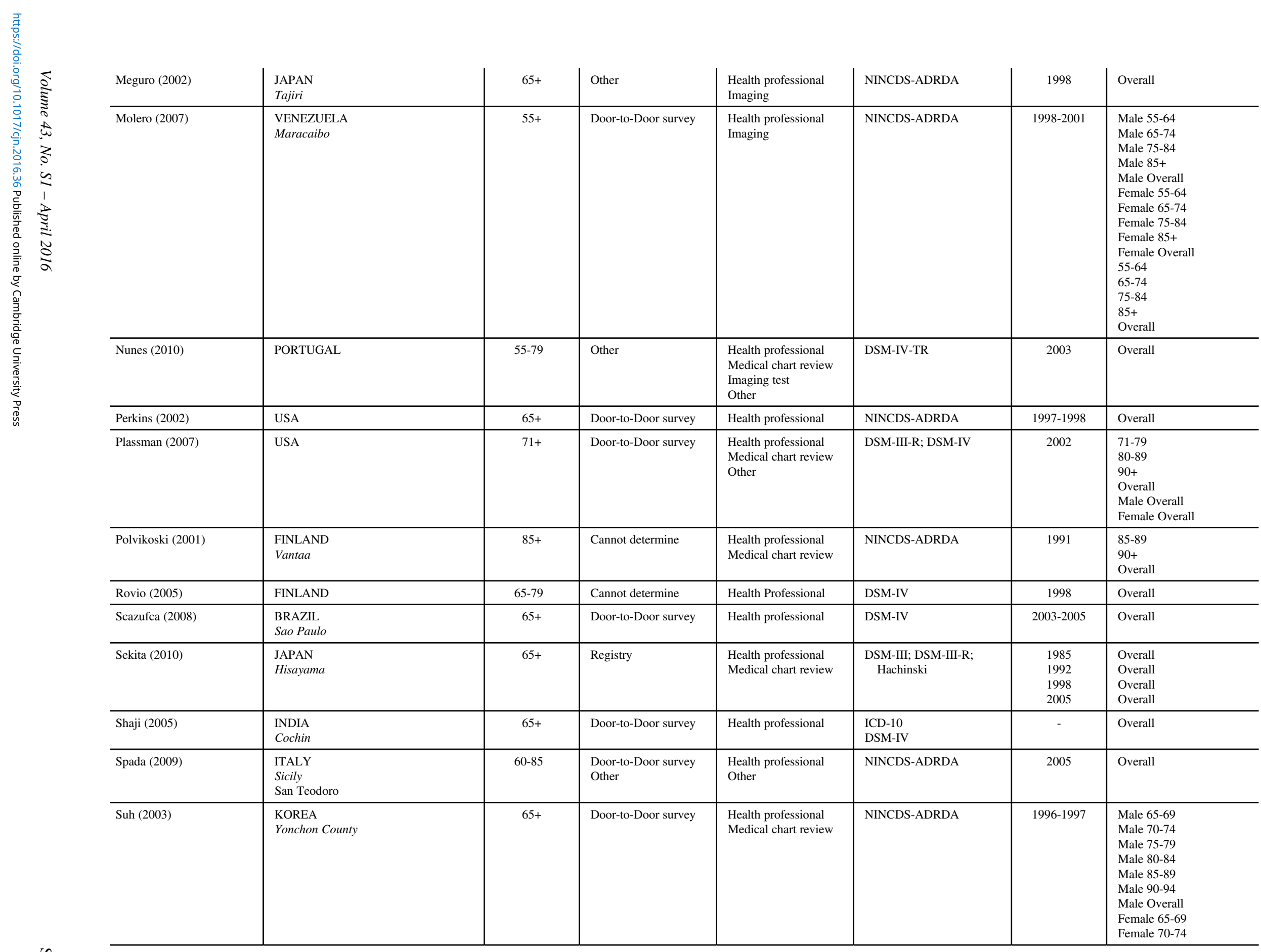


Table 1. (Continued)

\begin{tabular}{|c|c|c|c|c|c|c|c|}
\hline Author, Date & Country and Region & $\begin{array}{l}\text { Age Range } \\
\text { Studied }\end{array}$ & Data Source & $\begin{array}{l}\text { Diagnosis } \\
\text { Established by }\end{array}$ & Diagnostic Criteria & $\begin{array}{l}\text { Years of Data } \\
\text { Collection }\end{array}$ & Groups Studied \\
\hline & & & & & & & $\begin{array}{l}\text { Female } 75-79 \\
\text { Female } 80-84 \\
\text { Female } 85-89 \\
\text { Female 90-94 } \\
\text { Female Overall } \\
65-69 \\
70-74 \\
75-79 \\
80-84 \\
85-89 \\
90-94 \\
\text { Overall }\end{array}$ \\
\hline Vanhanen (2006) & $\begin{array}{l}\text { FINLAND } \\
\text { Kuоріо }\end{array}$ & $69-78$ & Cannot determine & $\begin{array}{l}\text { Health Professional } \\
\text { Imaging }\end{array}$ & NINCDS-ADRDA & $1990-1991$ & Overall \\
\hline Vas (2001) & $\begin{array}{l}\text { INDIA } \\
\text { Bombay }\end{array}$ & $40+$ & $\begin{array}{l}\text { Door-to-Door survey } \\
\text { Mailed survey } \\
\text { Other }\end{array}$ & $\begin{array}{l}\text { Health professional } \\
\text { Imaging test }\end{array}$ & NINCDS-ADRDA & 1991 & Overall \\
\hline Wada-Isoe (2009) & $\begin{array}{l}\text { JAPAN } \\
\text { Amino-Cho }\end{array}$ & $65+$ & Door-to-Door survey & $\begin{array}{l}\text { Health professional } \\
\text { Imaging }\end{array}$ & NINCDS-ADRDA & 2008 & $\begin{array}{l}65-69 \\
70-74 \\
75-79 \\
80-84 \\
85-89 \\
90+ \\
\text { Overall } \\
\text { Male 65-69 } \\
\text { Male 70-74 } \\
\text { Male 75-79 } \\
\text { Male 80-84 } \\
\text { Male 85-89 } \\
\text { Male 90+ } \\
\text { Male Overall } \\
\text { Female 65-69 } \\
\text { Female 70-74 } \\
\text { Female 75-79 } \\
\text { Female 80-84 } \\
\text { Female 85-89 } \\
\text { Female 90+ } \\
\text { Female Overall }\end{array}$ \\
\hline Wakutani (2007) & $\begin{array}{l}\text { JAPAN } \\
\text { Daisen-Cho }\end{array}$ & $65+$ & $\begin{array}{l}\text { Hospital/ clinic chart } \\
\text { review } \\
\text { Administrative } \\
\text { databases }\end{array}$ & Health professional & DSM-III; Hachinski & $\begin{array}{l}1980 \\
1990 \\
2000\end{array}$ & $\begin{array}{l}\text { Overall } \\
\text { Overall } \\
\text { Overall }\end{array}$ \\
\hline Wangtongkum (2008) & $\begin{array}{l}\text { THAILAND } \\
\text { Chian Mai province }\end{array}$ & $45+$ & Door-to-Door survey & $\begin{array}{l}\text { Health professional } \\
\text { Imaging test } \\
\text { Other }\end{array}$ & DSM-IV & 2004-2005 & Overall \\
\hline Xu (2009) & SWEDEN & $65+$ & $\begin{array}{l}\text { Registry } \\
\text { Telephone survey }\end{array}$ & Health professional & NINCDS-ADRDA & 1998-2001 & Overall \\
\hline Yamada (2001) & $\begin{array}{l}\text { JAPAN } \\
\text { Amino-Cho }\end{array}$ & $65+$ & Door-to-Door survey & $\begin{array}{l}\text { Health professional } \\
\text { Imaging test } \\
\text { Other }\end{array}$ & NINCDS-ADRDA & 1998 & $\begin{array}{l}\text { Male Overall } \\
\text { Female Overall } \\
\text { Overall }\end{array}$ \\
\hline
\end{tabular}


Table 1. (Continued)

\begin{tabular}{|c|c|c|c|c|c|c|c|}
\hline Author, Date & Country and Region & $\begin{array}{l}\text { Age Range } \\
\text { Studied }\end{array}$ & Data Source & $\begin{array}{l}\text { Diagnosis } \\
\text { Established by }\end{array}$ & Diagnostic Criteria & $\begin{array}{l}\text { Years of Data } \\
\text { Collection }\end{array}$ & Groups Studied \\
\hline \multicolumn{8}{|l|}{ Community \& Institution } \\
\hline Arslantas (2009) & $\begin{array}{l}\text { TURKEY } \\
\text { Eskisehir }\end{array}$ & $55+$ & Door-to-Door survey & $\begin{array}{l}\text { Health professional } \\
\text { Imaging test }\end{array}$ & NINCDS-ADRDA & $2002-2004$ & Overall \\
\hline Benedetti (2002) & ITALY & $75+$ & Door-to-door survey & Health professional & NINCDS-ADRDA & 1996 & $\begin{array}{l}\text { Female 75-79 } \\
\text { Female 80-84 } \\
\text { Female 85-89 } \\
\text { Female 90-97 } \\
\text { Female Overall } \\
\text { Male 75-79 } \\
\text { Male 80-84 } \\
\text { Male 85-89 } \\
\text { Male 90-97 } \\
\text { Male Overall } \\
75-79 \\
80-84 \\
85-89 \\
90-97 \\
\text { Overall }\end{array}$ \\
\hline Borroni (2011) & ITALY & $45-65$ & Registry & $\begin{array}{l}\text { Health professional } \\
\text { Imaging } \\
\text { Other }\end{array}$ & None & 2009 & $\begin{array}{l}\text { Overall } \\
\text { Male Overall } \\
\text { Female Overall }\end{array}$ \\
\hline Camicioli (2000) & USA & $65+$ & $\begin{array}{l}\text { Registry } \\
\text { Chart Review }\end{array}$ & Medical Chart review & NINCDS-ADRDA & 1994 & Overall \\
\hline $\begin{array}{l}\text { Canadian Study of Health } \\
\text { and Aging Working } \\
\text { Group (1994) }\end{array}$ & CANADA & $65+$ & $\begin{array}{c}\text { Administrative } \\
\text { Databases }\end{array}$ & Health professional & NINCDS-ADRDA & 1991 & $\begin{array}{l}\text { Male 85+ } \\
\text { Female 85+ } \\
85+ \\
\text { Male 65-74 } \\
\text { Male 75-84 } \\
\text { Male 85+ } \\
\text { Male Overall } \\
\text { Female 65-74 } \\
\text { Female } 75-84 \\
\text { Female 85+ } \\
\text { Female Overall } \\
65-74 \\
75-84 \\
85+ \\
\text { Overall } \\
65-74 \\
75-84 \\
\end{array}$ \\
\hline Chen (2007) & TAIWAN & $65+$ & $\begin{array}{l}\text { Other: Nursing Home } \\
\text { Records }\end{array}$ & Health professional & NINCDS-ADRDA & 2004 & $\begin{array}{l}\text { Male Overall } \\
\text { Female Overall } \\
\text { Overall }\end{array}$ \\
\hline Ebly (1994) & CANADA & $85+$ & Other & Health professional & NINCDS-ADRDA & 1990-1992 & $\begin{array}{l}\text { Male } 85-89 \\
\text { Male 90-94 } \\
\text { Male } 95+ \\
\text { Male Overall } \\
\text { Female } 85-89 \\
\text { Female } 90-94\end{array}$ \\
\hline
\end{tabular}




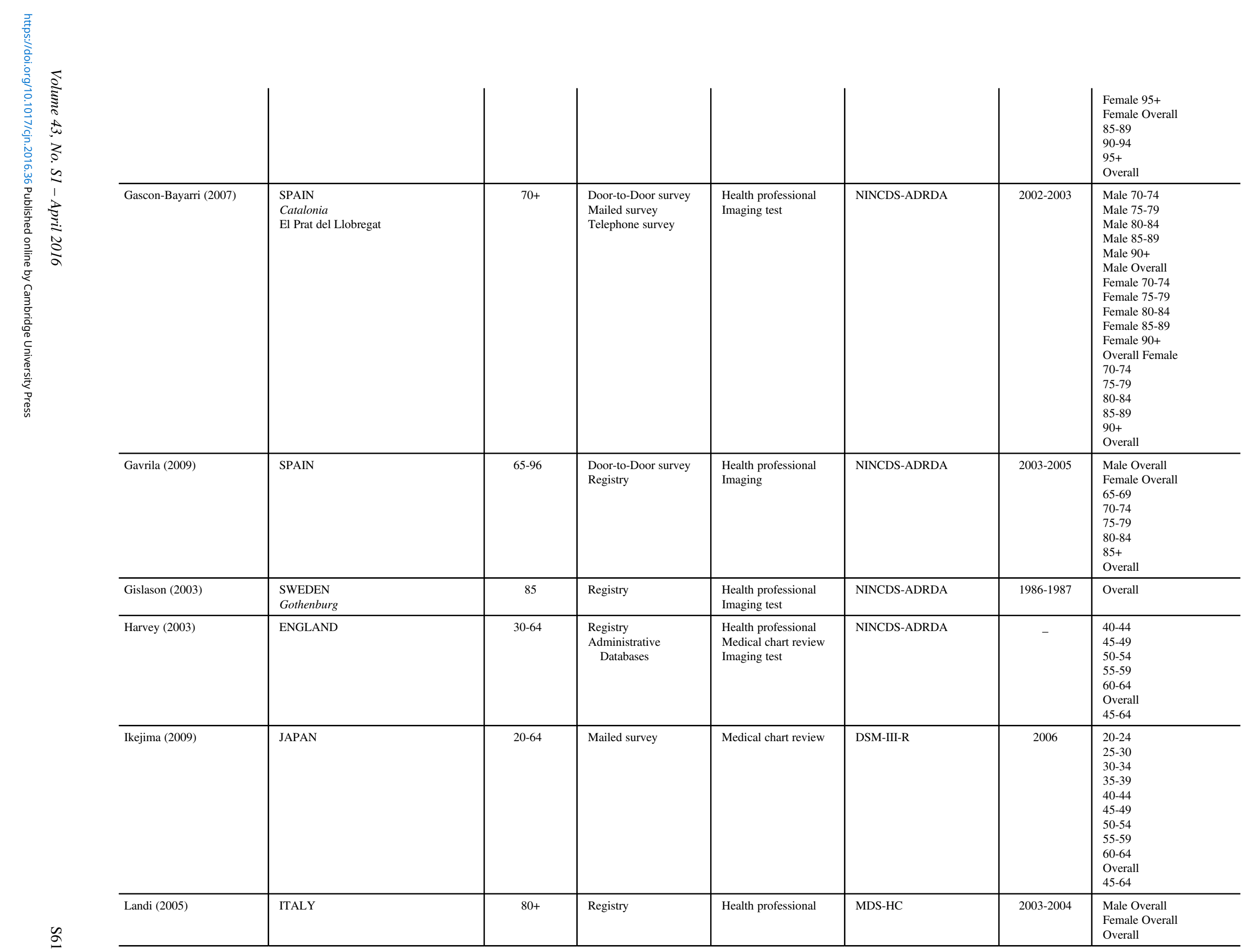


Table 1. (Continued)

\begin{tabular}{|c|c|c|c|c|c|c|c|}
\hline Author, Date & Country and Region & $\begin{array}{l}\text { Age Range } \\
\text { Studied }\end{array}$ & Data Source & $\begin{array}{l}\text { Diagnosis } \\
\text { Established by }\end{array}$ & Diagnostic Criteria & $\begin{array}{l}\text { Years of Data } \\
\text { Collection }\end{array}$ & Groups Studied \\
\hline Lovheim (2008) & SWEDEN & $85+$ & Other & $\begin{array}{l}\text { Health professional } \\
\text { Medical chart review } \\
\text { Other }\end{array}$ & DSM-IV & 2005-2006 & Overall \\
\hline Manton (2005) & USA & $65+$ & Registry & Cannot determine & SPMSQ or MMSE & 1982-1999 & $\begin{array}{l}\text { Overall } \\
65-79 \\
80+ \\
\text { Male Overall } \\
\text { Male 65-79 } \\
\text { Male 80+ } \\
\text { Female Overall } \\
\text { Female 65-79 } \\
\text { Female } 80+\end{array}$ \\
\hline Phung (2010) & DENMARK & $40+$ & Registry & $\begin{array}{l}\text { Administrative data } \\
\text { codes }\end{array}$ & ICD-8/10 & 1970-2004 & Overall \\
\hline Rahkonen (2003) & $\begin{array}{l}\text { FINLAND } \\
\text { Kuopio }\end{array}$ & $75+$ & Cannot determine & $\begin{array}{l}\text { Health professional } \\
\text { Medical chart review } \\
\text { Other }\end{array}$ & DSM-IV & 1998 & $\begin{array}{l}\text { Overall } \\
\text { Male Overall } \\
\text { Female Overall } \\
75-79 \\
80-84 \\
85-89 \\
90+\end{array}$ \\
\hline Rockwood (2000) & CANADA & $65+$ & Other & Health professional & NINCDS-ADRDA & $\begin{array}{l}\text { 1991-1992, } \\
1996\end{array}$ & $\begin{array}{l}65-74 \\
75-84 \\
85+ \\
\text { Overall } \\
\text { Male Overall } \\
\text { Female Overall }\end{array}$ \\
\hline Sahadevan (2008) & SINGAPORE & $50+$ & Door-to-Door survey & $\begin{array}{l}\text { Health professional } \\
\text { Other }\end{array}$ & NINCDS-ADRDA & 2001-2003 & $\begin{array}{l}\text { Overall } \\
\text { Male Overall } \\
\text { Female Overall } \\
50-59 \\
60-69 \\
70-79 \\
80+\end{array}$ \\
\hline Stevens (2002) & $\begin{array}{l}\text { UK } \\
\text { London } \\
\text { Islington }\end{array}$ & $65+$ & Door-to-Door survey & $\begin{array}{l}\text { Health professional } \\
\text { Medical chart review } \\
\text { Other }\end{array}$ & $\begin{array}{l}\text { NINCDS-ADRDA } \\
\text { DSM-IV }\end{array}$ & - & Overall \\
\hline Zhang (2005) & CHINA & $55+$ & $\begin{array}{l}\text { Door-to-Door survey } \\
\text { Census }\end{array}$ & Health professional & NINCDS-ADRDA & 1997 & $\begin{array}{l}\text { Male 55-64 } \\
\text { Male 65-74 } \\
\text { Male 75-84 } \\
\text { Male 85+ } \\
\text { Male Overall } \\
\text { Female 55-64 } \\
\text { Female 65-74 } \\
\text { Female 75-84 } \\
\text { Female } 85+ \\
\text { Female Overall } \\
\text { 55-64 } \\
65-74 \\
75-84 \\
85+ \\
\text { Overall }\end{array}$ \\
\hline
\end{tabular}




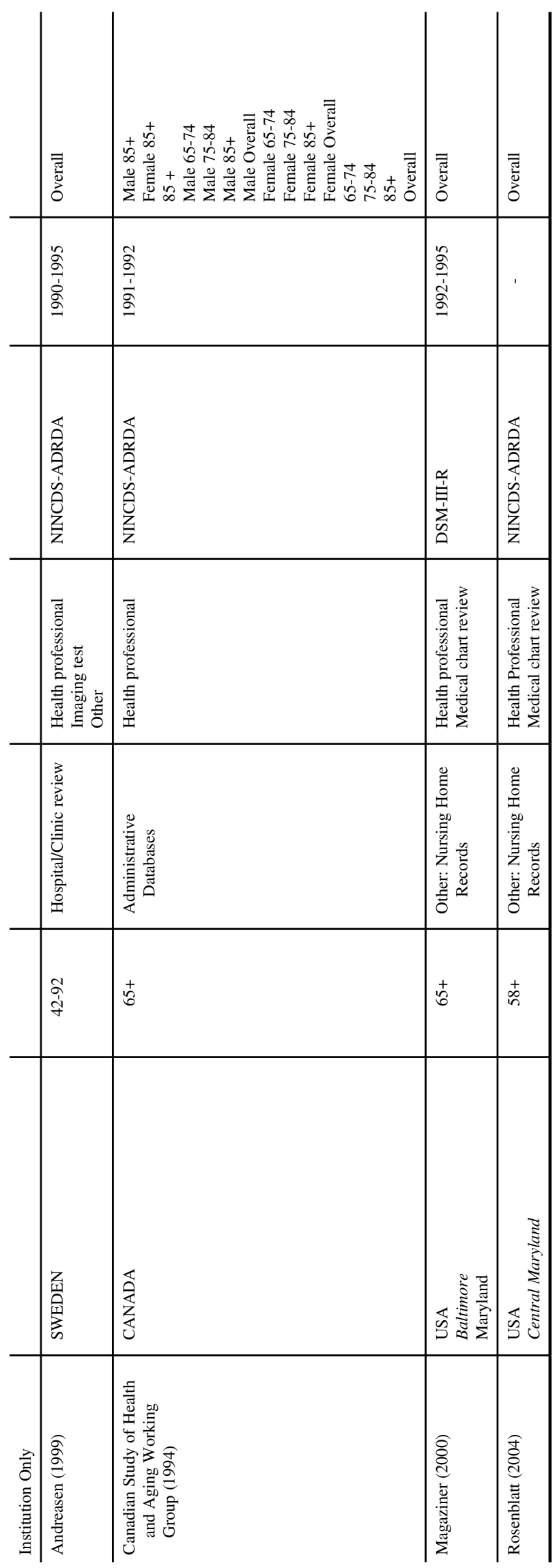

36 from Asia, 32 from North America, 5 from South America, 2 from Africa and 2 from Australia (2 studies reported data from more than one continent). Nine studies reported on those aged $60+, 68$ on those $65+$ and 19 on those $70+$.

\section{Prevalence of AD}

Forty-five articles ${ }^{28-72}$ reported on the point prevalence of $\mathrm{AD}$ dementia, with 20 eligible for inclusion in the meta-analysis of those aged $60+.^{28,29,31,33,35,36,38,39,42,45,46,48,57,60-63,65,67,70}$ In community-only settings, the point prevalence among those $60+$ years of age was $40.19\left(\mathrm{CI}_{95 \%}: 29.06-55.59\right)$ per 1000 (Figure 2). Point prevalence estimates in the community ranged from a low of 15.51 per 1000 in one study from India ${ }^{60}$ to a high of 204.13 per 1000 in a study from Israel. ${ }^{70}$ The pooled point prevalence in those $60+$ in combined community and institution studies was $26.57\left(\mathrm{CI}_{95 \%}\right.$ : $\left.11.83-59.69\right)$ per 1000 . In community and institution studies, point prevalence estimates ranged from a low of 12.34 per 1000 in a study from the United States ${ }^{35}$ to a high of 51.00 per 1000 in a study from Canada. ${ }^{28}$ The pooled point prevalence of $\mathrm{AD}$ among those $60+$ in institution-only settings was $226.97\left(\mathrm{CI}_{95 \%}\right.$ : 88.23-583.87).

Thirty studies reported on the period prevalence of $\mathrm{AD},{ }^{73-103}$ with 10 eligible for inclusion in the meta-analysis of those $60+.{ }^{77,82,83,87,89-91,99,100,102}$ In community settings, the pooled annual period prevalence among those aged $60+$ was 30.4 (CI ${ }_{95 \%}$ : 15.6-59.1) per 1000 (Figure 3 ). In combined community and institution settings, the pooled annual period prevalence was 44.0 (CI $_{95 \%}$ : 19.9-97.1) per 1000. A single study from an institution in the United States reported an annual period prevalence of $101.0\left(\mathrm{CI}_{95 \%}\right.$ : 89.4-114.1) per $1000 .{ }^{93}$ Annual period prevalence estimates for any setting ranged from 1.1 per 1000 in a community study from India ${ }^{77}$ to 123.0 in a community study from the United States. $^{91}$

\section{Incidence of $\mathrm{AD}$}

Fourteen studies reported on the incidence proportion of $\mathrm{AD},{ }^{104-117}$ with six included in the meta-analysis of $60+$ studies. ${ }^{107,108,111,113,114,116}$ In community settings, the pooled annual incidence proportion among those aged $60+$ was 34.1 (CI ${ }_{95 \%}$ : 16.4-70.9) per 1000 (Figure 4). A single U.S. study reported on the annual incidence proportion in combined community and institution settings with an estimate of $27.2\left(\mathrm{CI}_{95 \%}: 22.2-33.3\right)$ per $1000{ }^{105}$ There were no studies from an institution-only setting. Annual incidence proportion estimates for any setting ranged from 11.5 per 1000 in a community study from Nigeria ${ }^{108}$ to 97.8 per 1000 in a community study from the United States. ${ }^{11}$

Thirty studies reported on the incidence rate of $\mathrm{AD},{ }^{72,118-146}$ with 11 eligible for inclusion in the meta-analysis of those $60+.^{118,122,126-129,136,140,143-145}$ In community-only settings, the pooled incidence rate of $\mathrm{AD}$ among those $60+$ was $15.8\left(\mathrm{CI}_{95 \%}\right.$ : 12.9-19.4) per 1000 person-years (Figure 5). A single Italian study reported on the incidence rate in combined community and institution settings with an estimate of $7.0\left(\mathrm{CI}_{95 \%}\right.$ : 5.5-8.9) per 1000 person-years. ${ }^{120}$ There were no studies from an institution-only setting. The lowest estimate for any setting was $7.0\left(\mathrm{CI}_{95 \%}\right.$ : 4.8-10.3) per 1000 person-years in a community study from the Netherlands ${ }^{145}$ and the aforementioned study from Italy, and the highest $30.0\left(\mathrm{CI}_{95 \%}\right.$ : 25.4-35.5) per 1000 person-years in a community study from the United States. ${ }^{143}$ 


\begin{tabular}{|c|c|c|c|c|c|c|c|c|}
\hline \multicolumn{9}{|c|}{ Table 2: Studies Reporting on the Incidence Rate of Alzheimer's Disease } \\
\hline & Author, Date & Country and Region & $\begin{array}{l}\text { Age Range } \\
\text { Studied }\end{array}$ & Data Source & $\begin{array}{l}\text { Diagnosis } \\
\text { Established by }\end{array}$ & Diagnostic Criteria & $\begin{array}{l}\text { Years of Data } \\
\text { Collection }\end{array}$ & Groups Studied \\
\hline & \multicolumn{8}{|l|}{ Community Only } \\
\hline & Bermejo-Pareja (2008) & $\begin{array}{l}\text { SPAIN } \\
\text { Las Margaritas, Lista, Arevalo }\end{array}$ & $65+$ & $\begin{array}{l}\text { Door-to-Door survey } \\
\text { Mailed survey }\end{array}$ & $\begin{array}{l}\text { Health professional } \\
\text { Administrative data } \\
\text { codes } \\
\text { Medical chart review }\end{array}$ & NINCDS-ADRDA & 1997-1998 & $\begin{array}{l}\text { Male 65-69 } \\
\text { Male 70-74 } \\
\text { Male e } 57-99 \\
\text { Male 80-84 } \\
\text { Male 85-90 } \\
\text { Male e+ } \\
\text { Male Overall } \\
\text { Female 65-69 } \\
\text { Female } 70-74 \\
\text { Female } 75-79 \\
\text { Female 80-84 } \\
\text { Female } 85-90 \\
\text { Female } 90+ \\
\text { Female Overall } \\
65-69 \\
70-74 \\
75-79 \\
80-84 \\
85-90 \\
90+ \\
\text { Overall }\end{array}$ \\
\hline & Chandra (2001) & INDIA & $55+$ & $\begin{array}{l}\text { Door-to-Door survey } \\
\text { Registry }\end{array}$ & Health professional & NINCDS-ADRDA & - & $\begin{array}{l}\text { Male 55-64 } \\
\text { Male 65-74 } \\
\text { Male 75-84 } \\
\text { Male 85+ } \\
\text { Male 65+ } \\
\text { Male Overall } \\
\text { Female 55-64 } \\
\text { Female 65-74 } \\
\text { Female 75-84 } \\
\text { Female 85+ } \\
\text { Female 55+ } \\
65+ \\
55-64 \\
65-74 \\
75-84 \\
85+ \\
\text { Overall }\end{array}$ \\
\hline & Fitzpatrick (2004) & USA & $65+$ & Door-to-Door survey & $\begin{array}{l}\text { Health professional } \\
\text { Imaging }\end{array}$ & NINCDS-ADRDA & 1992-1994 & $\begin{array}{l}\text { Overall } \\
<75 \\
75-79 \\
80-84 \\
85+ \\
\end{array}$ \\
\hline & Fuhrer (2003) & $\begin{array}{l}\text { FRANCE } \\
\text { Gironde and Dordogne }\end{array}$ & $65+$ & $\begin{array}{l}\text { Door-to-Door survey } \\
\text { Registry }\end{array}$ & $\begin{array}{l}\text { Health professional } \\
\text { Imaging test }\end{array}$ & NINCDS-ADRDA & 1988-1997 & Overall \\
\hline & Kukull (2002) & $\begin{array}{l}\text { USA } \\
\text { Washington } \\
\text { Seattle }\end{array}$ & $65+$ & $\begin{array}{l}\text { Door-to-Door survey } \\
\text { Other }\end{array}$ & $\begin{array}{l}\text { Health professional } \\
\text { Imaging test } \\
\text { Other }\end{array}$ & NINCDS-ADRDA & 1994 & $\begin{array}{l}65-69 \\
77-74 \\
75-79 \\
80-84 \\
85-89 \\
90+ \\
\text { Overall }\end{array}$ \\
\hline
\end{tabular}


Table 2. (Continued)

\begin{tabular}{|c|c|c|c|c|c|c|c|}
\hline Author, Date & Country and Region & $\begin{array}{c}\text { Age Range } \\
\text { Studied }\end{array}$ & Data Source & $\begin{array}{l}\text { Diagnosis } \\
\text { Established by }\end{array}$ & Diagnostic Criteria & $\begin{array}{l}\text { Years of Data } \\
\text { Collection }\end{array}$ & Groups Studied \\
\hline & & & & & & & $\begin{array}{l}65-74 \\
75-84 \\
85-94 \\
\text { Overall }\end{array}$ \\
\hline Ravalglia (2008) & $\begin{array}{l}\text { ITALY } \\
\text { Conselice } \\
\text { Ravenna } \\
\text { Emilia Romagna region }\end{array}$ & $65+$ & $\begin{array}{l}\text { Door-to-Door survey } \\
\text { Registry }\end{array}$ & $\begin{array}{l}\text { Health professional } \\
\text { Medical chart review } \\
\text { Imaging test }\end{array}$ & NINCDS-ADRDA & 1999-2004 & Overall \\
\hline Tang (2001) & USA & $65+$ & $\begin{array}{l}\text { Administrative } \\
\text { databases }\end{array}$ & $\begin{array}{l}\text { Health professional } \\
\text { Imaging test }\end{array}$ & NINCDS-ADRDA & 1992-1999 & $\begin{array}{l}65-74 \\
75-84 \\
85+ \\
\text { Overall }\end{array}$ \\
\hline Tyas (2006) & $\begin{array}{l}\text { CANADA } \\
\text { Manitoba }\end{array}$ & $65+$ & $\begin{array}{l}\text { Registry } \\
\text { Administrative } \\
\text { databases }\end{array}$ & $\begin{array}{l}\text { Health professional } \\
\text { Other }\end{array}$ & NINCDS-ADRDA & 1991-1997 & $\begin{array}{l}\text { Male 65-69 } \\
\text { Male 70-74 } \\
\text { Male 75-79 } \\
\text { Male 80-84 } \\
\text { Male 85-89 } \\
\text { Male 90+ } \\
\text { Male Overall } \\
\text { Female 65-69 } \\
\text { Female 70-74 } \\
\text { Female 75-79 } \\
\text { Female 80-84 } \\
\text { Female } 85-89 \\
\text { Female 90+ } \\
65-69 \\
70-74 \\
75-79 \\
80-84 \\
85-89 \\
90+ \\
\text { Overall }\end{array}$ \\
\hline Waite (2001) & $\begin{array}{l}\text { AUSTRALIA } \\
\text { Sydney }\end{array}$ & $75+$ & $\begin{array}{l}\text { Door-to-Door survey } \\
\text { Census }\end{array}$ & Health professional & NINCDS-ADRDA & 1991-1994 & $\begin{array}{l}\text { Female } 75-79 \\
\text { Female } 80-84 \\
\text { Female } 85-89 \\
\text { Female 90+ } \\
\text { Female Overall } \\
\text { Male 75-79 } \\
\text { Male 80-84 } \\
\text { Male 85-89 } \\
\text { Male 90+ } \\
\text { Male Overall } \\
75-79 \\
80-84 \\
85-89 \\
90+ \\
\text { Overall }\end{array}$ \\
\hline
\end{tabular}




\begin{tabular}{|c|c|c|c|c|c|c|c|}
\hline \multicolumn{8}{|c|}{ Community \& Institution } \\
\hline Di Carlo (2002) & $\begin{array}{l}\text { ITALY } \\
\text { Genoa, Segrate (Milan), Selvazzano- } \\
\text { Rubano (Padua), Impruneta } \\
\text { (Florence), Fermo (Ascoli Piceno), } \\
\text { Naples, Casamassima (Bari), and } \\
\text { Catania }\end{array}$ & $65-84$ & $\begin{array}{l}\text { Door-to-Door survey } \\
\text { Registry }\end{array}$ & $\begin{array}{l}\text { Health professional } \\
\text { Medical chart review }\end{array}$ & NINCDS-ADRDA & 1995 & $\begin{array}{l}\text { Male 65-69 } \\
\text { Male 70-74 } \\
\text { Male 75-79 } \\
\text { Male 80-84 } \\
\text { Male Overall } \\
\text { Female 65-69 } \\
\text { Female 70-74 } \\
\text { Female 75-79 } \\
\text { Female 80-84 } \\
\text { Female Overall } \\
65-69 \\
70-74 \\
75-79 \\
80-84 \\
\text { Overall }\end{array}$ \\
\hline Edland (2002) & $\begin{array}{l}\text { UNITED STATES } \\
\text { Minnesota } \\
\text { Rochester }\end{array}$ & $50+$ & $\begin{array}{l}\text { Hospital/Clinic chart } \\
\text { review } \\
\text { Administrative } \\
\text { Databases }\end{array}$ & Medical chart review & DSM-IV & 1985-1989 & $\begin{array}{l}\text { Female 50-54 } \\
\text { Female 55-59 } \\
\text { Female 60-64 } \\
\text { Female 65-69 } \\
\text { Female 70-74 } \\
\text { Female 75-79 } \\
\text { Female 80-84 } \\
\text { Female 85-89 } \\
\text { Female 90-94 } \\
\text { Female 95-99 } \\
\text { Female Overall } \\
\text { Male 50-54 } \\
\text { Male 55-59 } \\
\text { Male 60-64 } \\
\text { Male 65-69 } \\
\text { Male 70-74 } \\
\text { Male 75-79 } \\
\text { Male 80-84 } \\
\text { Male 85-89 } \\
\text { Male 90-94 } \\
\text { Male 95-99 } \\
\text { Male Overall } \\
\text { 50-54 } \\
55-59 \\
60-64 \\
65-69 \\
70-74 \\
75-79 \\
80-84 \\
85-89 \\
90-94 \\
95-99 \\
\text { Overall }\end{array}$ \\
\hline Garre-Olmo (2010) & $\begin{array}{l}\text { SPAIN } \\
\text { Catolonia }\end{array}$ & $30-64$ & Registry & $\begin{array}{l}\text { Health professional } \\
\text { Medical chart review } \\
\text { Imaging test }\end{array}$ & DSM-IV-TR & 2007-2009 & $\begin{array}{l}30-64 \\
65+ \\
\text { Overall }\end{array}$ \\
\hline Knopman (2004) & USA & $40-70$ & $\begin{array}{l}\text { Administrative } \\
\text { Databases }\end{array}$ & Medical chart review & DSM-IV & 1990-1994 & $\begin{array}{l}40-49 \\
50-59 \\
60-69\end{array}$ \\
\hline
\end{tabular}


Table 2. (Continued)

\begin{tabular}{|c|c|c|c|c|c|c|c|}
\hline Author, Date & Country and Region & $\begin{array}{l}\text { Age Range } \\
\text { Studied }\end{array}$ & Data Source & $\begin{array}{l}\text { Diagnosis } \\
\text { Established by }\end{array}$ & Diagnostic Criteria & $\begin{array}{l}\text { Years of Data } \\
\text { Collection }\end{array}$ & Groups Studied \\
\hline McDowell (2007) & CANADA & $65+$ & $\begin{array}{l}\text { Other: Canadian Study } \\
\text { of Health and Aging }\end{array}$ & Health professional & NINCDS-ADRDA & 1991-2001 & Overall \\
\hline Ruitenberg (2001) & $\begin{array}{l}\text { NETHERLANDS } \\
\text { Rotterdam } \\
\text { Ommoord }\end{array}$ & $55+$ & Door-to-Door survey & $\begin{array}{l}\text { Health professional } \\
\text { Medical chart review } \\
\text { Imaging test }\end{array}$ & NINCDS-ADRDA & 1990-1999 & $\begin{array}{l}\text { Female 55-59 } \\
\text { Female 60-64 } \\
\text { Female 65-69 } \\
\text { Female 70-74 } \\
\text { Female } 75-79 \\
\text { Female 80-84 } \\
\text { Female 85-89 } \\
\text { Female 90-94 } \\
\text { Female 95+ } \\
\text { Female Overall } \\
\text { Male 55-59 } \\
\text { Male 60-64 } \\
\text { Male 65-69 } \\
\text { Male 70-74 } \\
\text { Male 75-79 } \\
\text { Male 80-84 } \\
\text { Male 85-89 } \\
\text { Male 90-94 } \\
\text { Male 95+ } \\
\text { Male Overall } \\
55-59 \\
60-64 \\
65-69 \\
70-74 \\
75-79 \\
80-84 \\
85-89 \\
90-94 \\
95+ \\
\text { Overall }\end{array}$ \\
\hline
\end{tabular}




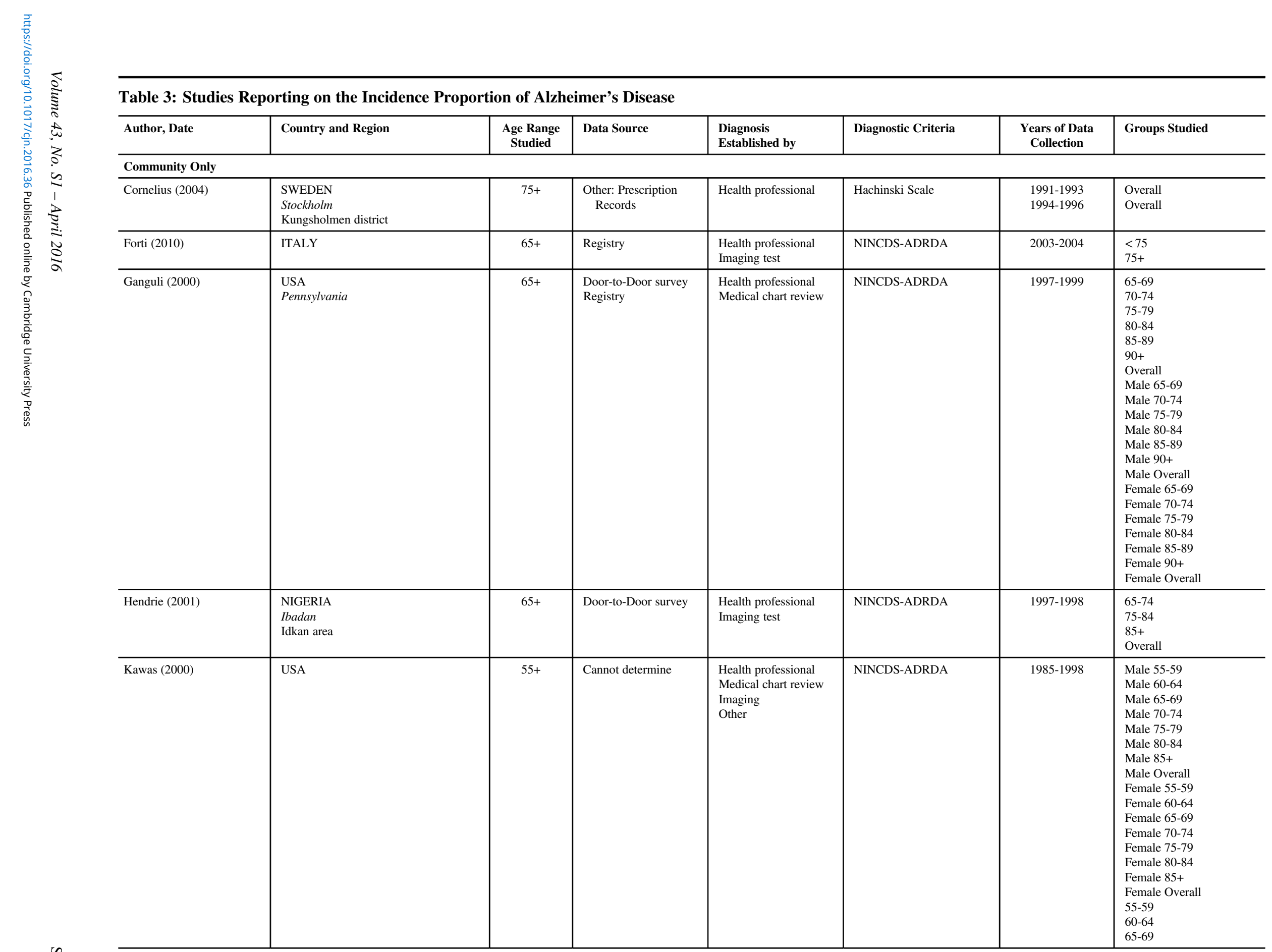


Table 3. (Continued)

\begin{tabular}{|c|c|c|c|c|c|c|c|}
\hline Author, Date & Country and Region & $\begin{array}{l}\text { Age Range } \\
\text { Studied }\end{array}$ & Data Source & $\begin{array}{l}\text { Diagnosis } \\
\text { Established by }\end{array}$ & Diagnostic Criteria & $\begin{array}{l}\text { Years of Data } \\
\text { Collection }\end{array}$ & Groups Studied \\
\hline & & & & & & & $\begin{array}{l}70-74 \\
75-79 \\
80-84 \\
85+ \\
\text { Overall }\end{array}$ \\
\hline Knopman (2003) & USA & $50-100$ & $\begin{array}{l}\text { Door-to-Door Survey } \\
\text { Registry }\end{array}$ & $\begin{array}{l}\text { Health professional } \\
\text { Imaging test }\end{array}$ & DSM-IV & $1985-1989$ & Overall \\
\hline Kuller (2005) & USA & $<70-80+$ & $\begin{array}{l}\text { Administrative } \\
\text { Databases }\end{array}$ & $\begin{array}{l}\text { Health professional } \\
\text { Medical chart review } \\
\text { Imaging test }\end{array}$ & NINCDS-ADRDA & 1998-1999 & Overall \\
\hline Lopez (2005) & USA & $65+$ & $\begin{array}{l}\text { Administrative } \\
\text { Databases }\end{array}$ & $\begin{array}{l}\text { Health professional } \\
\text { Medical chart review } \\
\text { Imaging test }\end{array}$ & NINCDS-ADRDA & 1994-1999 & Overall \\
\hline Lopez-Pousa (2004) & SPAIN & $75+$ & Door-to-Door survey & Health professional & DSM-II-R & $1990-1991$ & $\begin{array}{l}\text { Male 75-79 } \\
\text { Male 80-84 } \\
\text { Male } 85-89 \\
\text { Male 90+ } \\
\text { Male Overall } \\
\text { Female } 75-79 \\
\text { Female } 80-84 \\
\text { Female } 85-89 \\
\text { Female 90+ } \\
\text { Female Overall } \\
75-79 \\
80-84 \\
85-89 \\
\text { 90+ } \\
\text { Overall }\end{array}$ \\
\hline Miech (2002) & USA & $65+$ & Door-to-Door survey & $\begin{array}{l}\text { Health professional } \\
\text { Imaging test }\end{array}$ & NINCDS-ADRDA & 1998-1999 & $\begin{array}{l}\text { Overall } \\
\text { Male } \\
\text { Female }\end{array}$ \\
\hline Morris (2002) & USA & $65+$ & Census & $\begin{array}{l}\text { Health professional } \\
\text { Imaging test }\end{array}$ & NINCDS-ADRDA & $1993-2000$ & Overall \\
\hline Piguet (2003) & $\begin{array}{l}\text { AUSTRALIA } \\
\text { Sydney }\end{array}$ & $75+$ & Registry & Health professional & NINCDS-ADRDA & $1997-2000$ & Overall \\
\hline Seshadri (2002) & USA & $68-97$ & Other & $\begin{array}{l}\text { Health professional } \\
\text { Medical chart review } \\
\text { Imaging test }\end{array}$ & NINCDS-ADRDA & 1986-1990 & $\begin{array}{l}\text { Overall } \\
\text { Male Overall } \\
\text { Female Overall }\end{array}$ \\
\hline Vermeer (2003) & $\begin{array}{l}\text { NETHERLANDS } \\
\text { Rotterdam }\end{array}$ & $60-90$ & $\begin{array}{l}\text { Other: Previous survey } \\
\text { participants }\end{array}$ & $\begin{array}{l}\text { Health professional } \\
\text { Imaging test }\end{array}$ & NINCDS-ADRDA & $1999-2000$ & Overall \\
\hline Zandi (2002) & USA & $65+$ & Door-to-Door survey & $\begin{array}{l}\text { Health professional } \\
\text { Imaging test }\end{array}$ & DSM-III-R & $1998-2000$ & $\begin{array}{l}\text { Male Overall } \\
\text { Female Overall }\end{array}$ \\
\hline \multicolumn{8}{|c|}{ Community \& Institution } \\
\hline Evans (2003) & USA & $65+$ & Census & Health professional & NINCDS-ADRDA & - & Overall \\
\hline
\end{tabular}




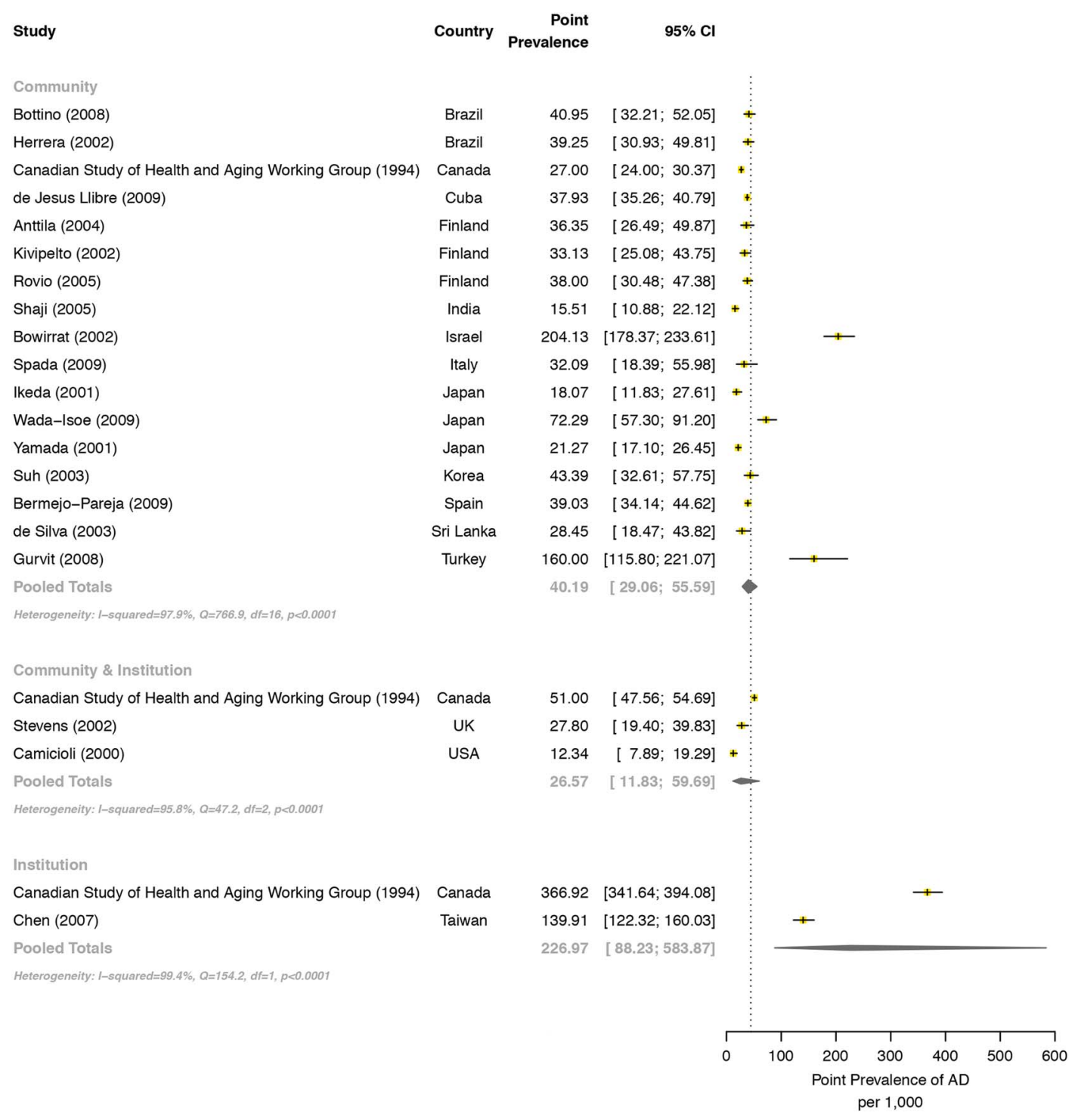

Figure 2: Pooled point prevalence of Alzheimer's Disease.

\section{Sources of Heterogeneity}

The effect of important potential sources of heterogeneity (i.e., age, sex, diagnostic criteria, location [continent], time [when the study was done]) on incidence and prevalence estimates in those aged $60+$ was assessed using univariate metaregressions.

\section{Age}

Increasing age was associated with increasing point prevalence, period prevalence, incidence rate and incidence proportion estimates $(p<0.001)$.

\section{Sex}

Though the differences did not reach statistical significance ( $p$ values ranged from 0.102 to 0.582 ), estimates of incidence and prevalence by sex of the subjects were higher in females than in males, in the 22 studies that reported on this.

\section{Diagnostic Criteria for AD}

Within community settings, DSM-IV criteria ${ }^{147}(n=2)$ produced a statistically significant $(p=0.044)$ higher estimate for AD dementia point prevalence (91.7 [CI $95 \%$ : 19.0-442.8] per 1000) than those based on NINCDS-ADRDA criteria for probable $\mathrm{AD}^{148}\left(n=14 ; 38.2 \quad\left[\mathrm{CI}_{95 \%}\right.\right.$ : 31.3-46.6] per 1000). No statistically significant differences between the aforementioned criteria were seen for period prevalence in the community $(p=0.065)$, though the association was in the same direction as seen in the pooled point prevalence. All incidence studies used NINCDS-ADRDA criteria for probable AD.

\section{Location}

Within community settings, the estimated annual period prevalence for North America $\left(n=2 ; 103.6\right.$ [CI ${ }_{95 \%}$ : 73.4-146.1] per 1000) was significantly higher than those for Asia $(n=4$; $11.7\left[\mathrm{CI}_{95 \%}: 2.8-48.5\right]$ per $\left.1000 ; p=0.017\right)$ and Europe $(n=2$; 31.3 [ $\mathrm{CI}_{95 \%}$ : 14.4-67.7] per $1000 ; p=0.006$ ). The estimates 


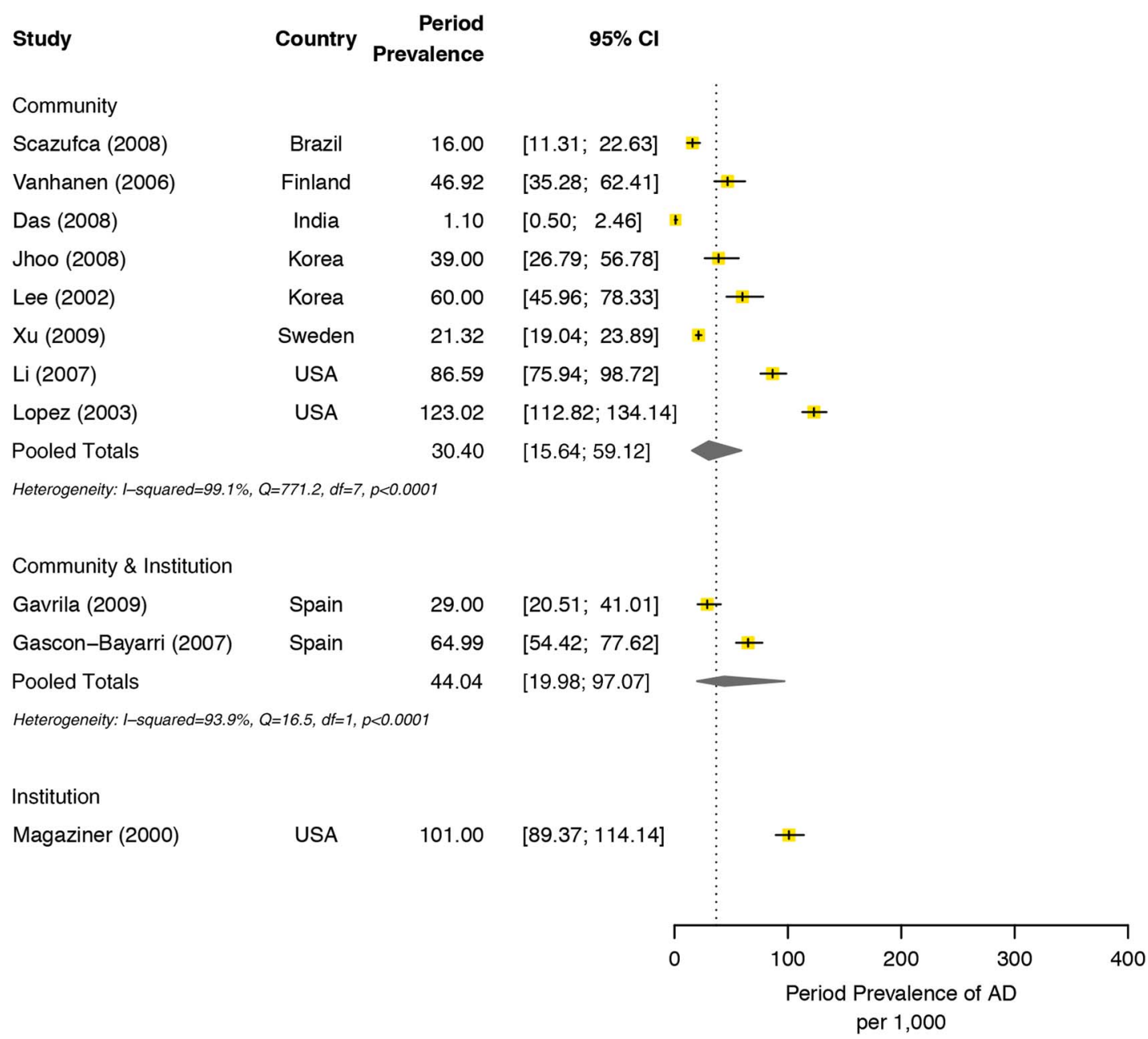

Figure 3: Pooled period prevalence of Alzheimer's disease.

for single studies for continents were: South America (16.0 $\left[\mathrm{CI}_{95 \%}: 11.3-22.6\right]$ per 1000) and Australia (88.0 [CI ${ }_{95 \%}$ : 82.7-93.7] per 1000). The incidence proportion estimate in a single-community African study (11.5 [ $\mathrm{CI}_{95 \%}$ : 9.70-13.64] per 1000) was lower than the estimated incidence proportion from five North American community studies (42.6 [ $\mathrm{CI}_{95 \%}$ : 23.0-78.8] per 1000) but could not be subjected to a meta-analysis as we required at least two estimates from a single region to be included.

\section{Time}

There was no effect of the time of study initiation, midpoint or conclusion on point prevalence, period prevalence, incidence rate or incidence proportion estimates.

\section{Publication Bias}

For the period prevalence, point prevalence, incidence rate and incidence proportion of $\mathrm{AD}$ dementia, significant funnel plot asymmetry was not found using Begg's or Egger's test $(p>0.05)$. Upon visual inspection, the funnel plots appeared symmetrical.

\section{Study Quality}

The median study quality score for studies reporting on the incidence or prevalence of $\mathrm{AD}$ dementia was 6/8 (range 3-8)
(Table 4). Study quality did not vary by continent based on the results of ANOVA analyses.

\section{DISCUSSION}

A substantial societal burden from AD dementia was demonstrated in our systematic review and meta-analyses. In community settings, the point prevalence of $\mathrm{AD}$ dementia among those $60+$ was 40.2 per 1000 , while its incidence proportion was 34.1 per 1000 and incidence rate was 15.8 per 1000 person-years. Despite the large number of studies included in our meta-analysis, the resulting estimates lacked precision at times due to significant statistical heterogeneity. Our finding that the period prevalence of $\mathrm{AD}$ dementia in community settings (30.4 per 1000 persons) was lower than the point prevalence (40.2 per 1000 persons) was unexpected and should be interpreted with caution. You would typically expect the opposite finding (i.e., a higher pooled estimate from the period prevalence studies). This was likely due to the significant heterogeneity (i.e., $>99 \%$ for period prevalence studies) that existed between these two pools of studies, leading to wide confidence intervals. In addition, there were several outliers, particularly in the period prevalence estimates, which ranged from a low of 1.1 in India $^{77}$ to a high of 123.0 per 1000 persons in a U.S. study. ${ }^{91}$ 


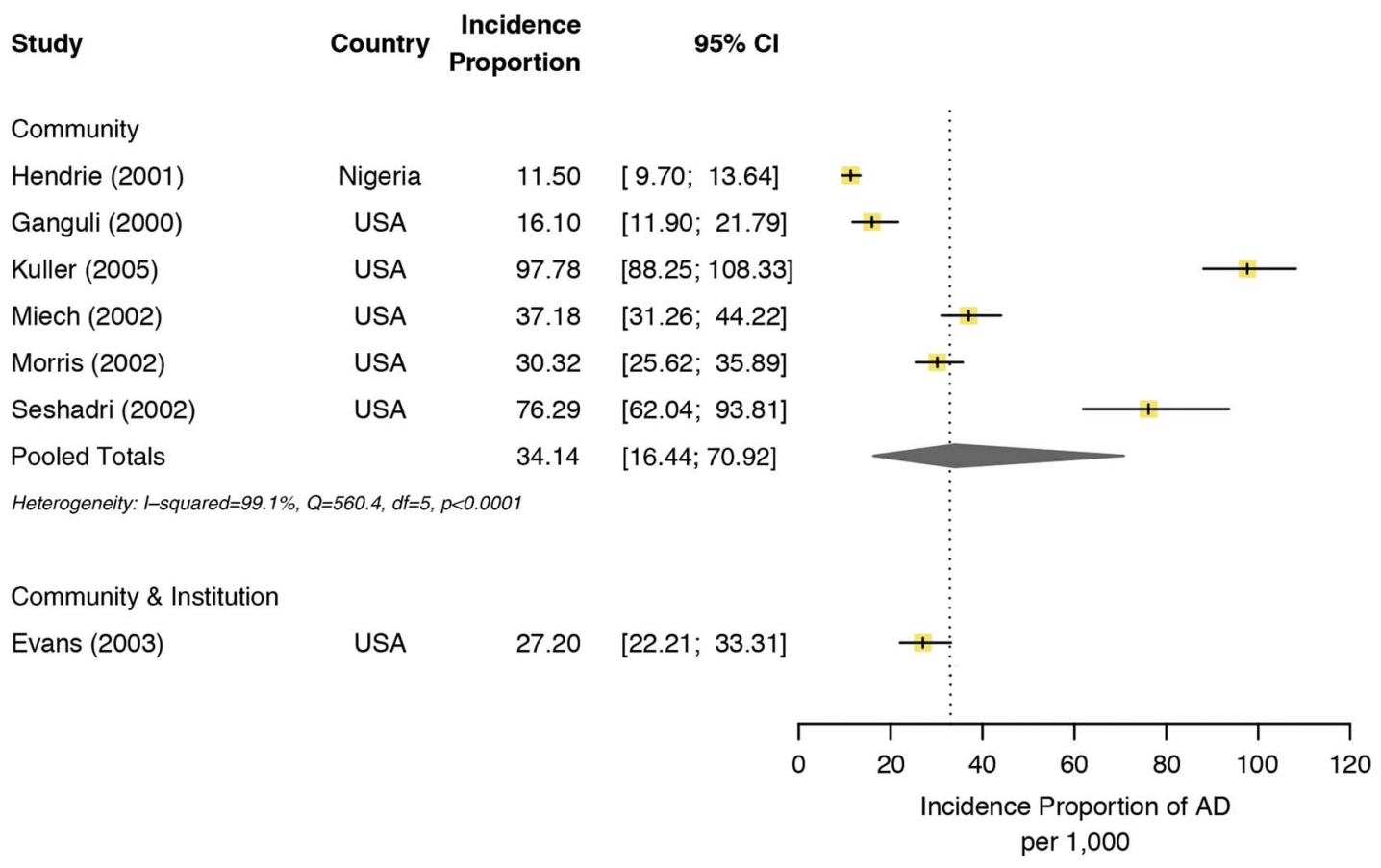

Figure 4: Pooled incidence proportion of Alzheimer's disease.

Our exploration of the sources of this heterogeneity led to several interesting findings. There was an insufficient number of population-based institution studies to do meta-analyses for this setting, but our descriptive analysis indicated that incidence rate for $\mathrm{AD}$ dementia is higher in the community while prevalence is greater in institutions. This is not surprising. There are few at-risk individuals within institutions, and the high mortality rate from other causes in the small at-risk institutional group likely means that they will likely die from another cause before they have time to develop AD. The inclusion of an institutionalized sample as well as region-specific variation in the availability of facility-based care and/or likelihood for institutionalization can have a substantial impact on the estimated prevalence of $\mathrm{AD}$ dementia in a community. ${ }^{149}$ Institutionalization typically occurs as a result of the functional impairments, behavioural challenges and associated burden on family caregivers that arise as the disease progresses and largely explain the high prevalence in this setting. Information on the incidence and prevalence of $\mathrm{AD}$ stratified by setting is particularly relevant for planning resource allocation. We identified a significant gap when it comes to the population-based epidemiology of AD dementia in institutional and residential settings. Future studies are required to understand the true burden of $\mathrm{AD}$ dementia in long-term and supportive care facilities. The use of standardized assessments based on data abstracted from interRAI instruments to provide estimates for the prevalence of dementia in these settings holds promise, but it is unclear whether they could be utilized for estimates of dementia arising specifically from $\mathrm{AD}^{150}$

All estimates of incidence and prevalence were higher for females compared to males, though the differences were not statistically different. In economically developed nations, about two-thirds of individuals diagnosed with $\mathrm{AD}$ dementia are women. ${ }^{151}$ This is primarily due to the fact that women on average live longer than men, and increasing age is the most important non-genetic risk factor for AD dementia. Incidence studies suggest an age-dependent relationship between sex and likelihood of developing AD dementia. One of the studies we included noted differences in incidence rates by sex after 90 years of age. ${ }^{142}$ Other reports indicate that the incidence of $\mathrm{AD}$ dementia increases with age in both sexes until 85-90 years of age, after which it plateaus for men but continues to increase among women. ${ }^{152,153} \mathrm{~A}$ prior meta-analysis reported slightly longer doubling times with increasing age for $\mathrm{AD}$ dementia in men compared to women, ${ }^{10}$ while another study reported that women tend to have a higher incidence at very advanced ages. ${ }^{12}$ These noted differences between the sexes could be due to methodological issues, the differential impact of historical environmental risk factors, or true biological differences in disease susceptibility between the sexes. ${ }^{154}$ Interestingly, recent data suggest that, relative to women, men who survive to older ages may exhibit a lower risk for developing $\mathrm{AD}$ because of a healthier cardiovascular risk profile. ${ }^{155}$

Difficulties examining the effect of the diagnostic criteria utilized to diagnose $\mathrm{AD}$ cases were encountered due to the ubiquitous use of NINCDS-ADRDA criteria. However, for point prevalence in community-only settings, DSM-IV criteria were found to produce significantly higher estimates than studies utilizing NINCDS-ADRDA criteria for probable AD (and possibly ICD-10 criteria ${ }^{156}$ ); a trend in the same direction was also shown in community period prevalence estimates. The choice and operationalization of diagnostic criteria can have a large effect on estimated incidence and/or prevalence. ${ }^{157,158}$ In one study, the use of DSM-III criteria led to $29.1 \%$ of subjects receiving a dementia diagnosis compared to $13.7 \%$ when DSM-IV criteria were employed. ${ }^{157}$ Newer diagnostic criteria for 


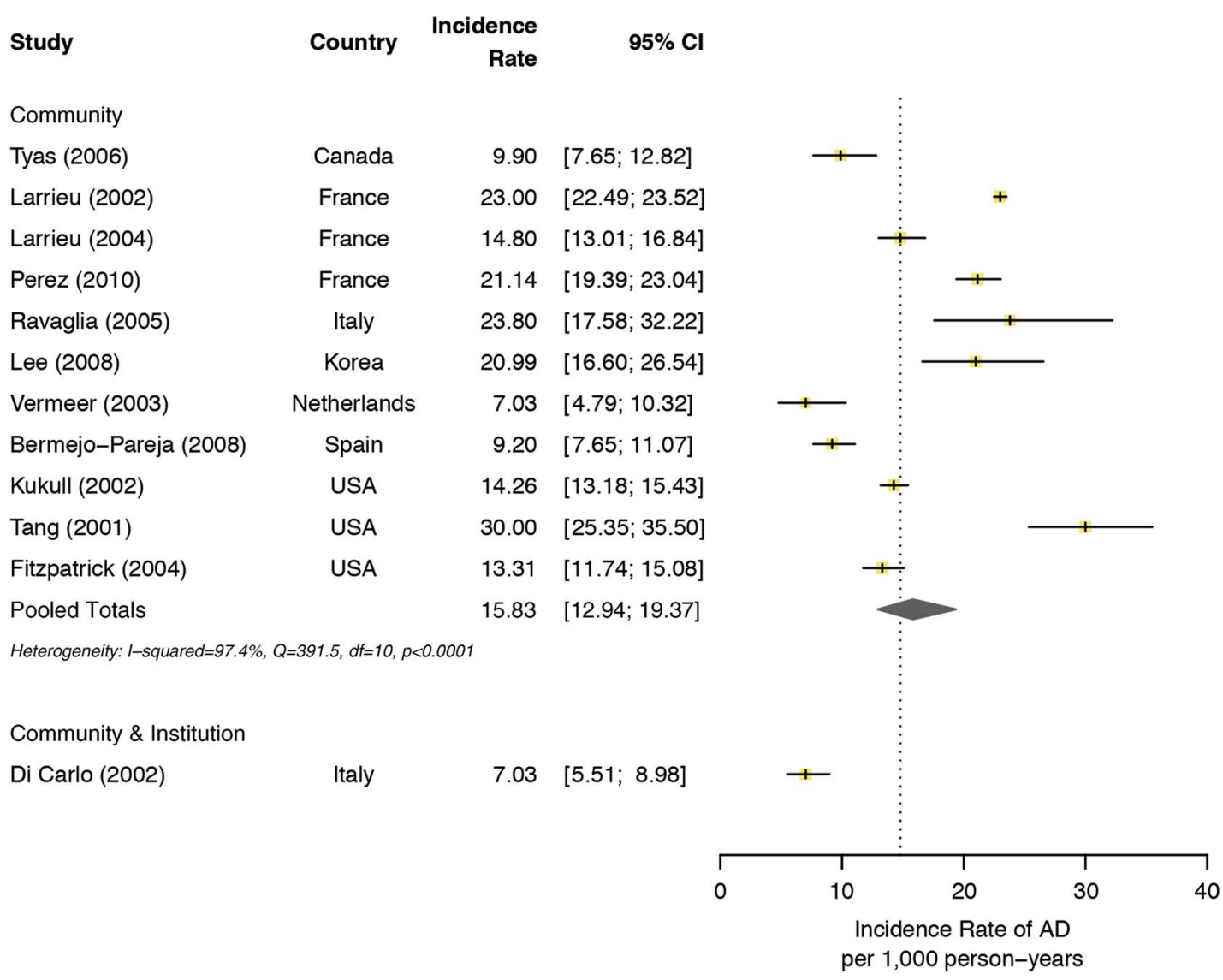

Figure 5: Pooled incidence rate of Alzheimer's disease.

$\mathrm{AD}$ decouple $\mathrm{AD}$ from the presence of a dementia and no longer require the presence of a memory impairment, the impact of which on epidemiological estimates of the incidence and prevalence of AD is yet unknown. ${ }^{159}$ In the future, a diagnosis of preclinical $\mathrm{AD}$ may be made on the basis of biomarkers, though clinical criteria will be required to diagnose symptomatic (i.e., mild cognitive impairment or dementia) $\mathrm{AD} .{ }^{160} \mathrm{We}$ suspect that the National Institute on Aging-Alzheimer's Association clinical diagnostic criteria for dementia due to $\mathrm{AD}^{161}$ will be used in future incidence and prevalence studies of AD. Studies are needed to assess the potential impact of using these newer diagnostic approaches compared to the criteria that have been used to date on the estimated incidence and prevalence of $\mathrm{AD}$ and trends over time.

Estimates of $\mathrm{AD}$ dementia incidence and prevalence tended to be higher in North America as compared to Asia, but these differences were not statistically different except for estimates of period prevalence in community settings. Geographical differences in epidemiological estimates of $\mathrm{AD}$ could be due to a variety of factors other than true differences in age-specific disease risk, such as differing screening methods and thresholds for diagnosis, age distribution of the assessed population, duration of survival after the onset of AD dementia, overall life expectancy and competing risks. ${ }^{17}$ Nonetheless, the possibility of true regional differences in $\mathrm{AD}$ incidence and prevalence has important implications. It is unlikely that the observed findings can be fully explained by differences in life expectancy. While North America has a relatively high life expectancy, estimates of life expectancy are similar or even higher in several Asian countries (e.g., Japan). ${ }^{162}$ Similar findings (i.e., lower estimates in Asia) have been reported for Huntington's disease and Parkinson's disease, where it is felt that differences in the distribution, life expectancy and degree of stigmatization associated with a diagnosis of the condition may contribute to variations in disease reporting. ${ }^{163,164}$

The methodology utilized for this systematic review and metaanalysis closely followed established guidelines. We feel we were able to identify most eligible studies as multiple sources of study ascertainment were employed. We found no evidence for publication bias. We did, however, find a good deal of statistical heterogeneity.

In order to accurately plan for future needs, there remains an ongoing requirement to provide accurate estimates of the incidence and prevalence of AD. Relying on older data may lead to either over- or underestimating the resources required if incidence and prevalence rates are changing over time. Though, using meta-regression analysis, we did not find that time had an effect on the incidence or prevalence of $\mathrm{AD}$, this does not preclude the possibility of true changes in age-standardized incidence and/or prevalence rates for dementia from $\mathrm{AD}$ occurring either now or in the near future due to changes in the presence of risk factors at a population level. ${ }^{165}$ There could well be rising and/or falling rates of $\mathrm{AD}$ within specific nations or regions that could be obscured by looking at international changes. For dementia overall, as an example, there is a suggestion that 
Table 4: Quality assessment scores of Alzheimer's Disease incidence and prevalence studies

\begin{tabular}{|c|c|c|c|c|c|c|c|c|c|}
\hline Study (Year) & $\begin{array}{l}\text { Q1: Target } \\
\text { population } \\
\text { described? }\end{array}$ & $\begin{array}{l}\text { Q2: Cases from entire } \\
\text { population or } \\
\text { probability sampling? }\end{array}$ & $\begin{array}{l}\text { Q3: } \\
\text { Response } \\
\text { rate > 70\%? }\end{array}$ & $\begin{array}{l}\text { Q4: Non- } \\
\text { responders } \\
\text { clearly described? }\end{array}$ & $\begin{array}{l}\text { Q5: Sample } \\
\text { representative } \\
\text { of population? }\end{array}$ & $\begin{array}{l}\text { Q6: Data collection } \\
\text { methods } \\
\text { standardized? }\end{array}$ & $\begin{array}{l}\text { Q7: Validated } \\
\text { criteria to } \\
\text { assess disease? }\end{array}$ & $\begin{array}{l}\text { Q8: Were estimates given } \\
\text { with confidence intervals } \\
\text { or subgroups? }\end{array}$ & $\begin{array}{l}\text { Total Quality } \\
\text { Score (/8) }\end{array}$ \\
\hline Andreasen (1999) & Yes & Yes & Yes & Yes & Yes & Yes & Yes & Yes & 8 \\
\hline Anttila (2004) & Yes & Yes & Yes & NR & NR & Yes & Yes & No & 5 \\
\hline Arslantas (2009) & Yes & Yes & $\mathrm{NC}$ & No & $\mathrm{NC}$ & Yes & Yes & Yes & 5 \\
\hline Banerjee (2008) & Yes & Yes & NR & NR & $\mathrm{NC}$ & Yes & Yes & Yes & 5 \\
\hline Benedetti (2002) & Yes & Yes & Yes & Yes & $\mathrm{NC}$ & Yes & Yes & Yes & 7 \\
\hline Bermejo-Pareja (2008) & Yes & Yes & Yes & Yes & $\mathrm{NC}$ & Yes & Yes & Yes & 7 \\
\hline Bermejo-Pareja (2009) & Yes & Yes & Yes & Yes & $\mathrm{NC}$ & Yes & Yes & Yes & 7 \\
\hline $\begin{array}{l}\text { Borjesson-Hanson } \\
\text { (2004) }\end{array}$ & Yes & Yes & No & No & $\mathrm{NC}$ & Yes & Yes & Yes & 5 \\
\hline Borroni (2011) & Yes & Yes & NR & NR & NR & Yes & Yes & Yes & 5 \\
\hline Bottino (2008) & Yes & No & No & No & $\mathrm{NC}$ & Yes & Yes & Yes & 4 \\
\hline Bowirrat (2001) & Yes & Yes & NR & Yes & Yes & No & No & No & 4 \\
\hline Bowirrat (2001) & Yes & Yes & NR & Yes & Yes & No & No & No & 4 \\
\hline Bowirrat (2001) & Yes & Yes & Yes & Yes & Yes & Yes & Yes & Yes & 8 \\
\hline Camicioli (2000) & Yes & Yes & Yes & Yes & $\mathrm{NC}$ & Yes & Yes & No & 6 \\
\hline $\begin{array}{l}\text { Canadian Study of } \\
\text { Health and Aging } \\
\text { Working Group } \\
\text { (1994) }\end{array}$ & Yes & Yes & Yes & NR & $\mathrm{NC}$ & Yes & Yes & Yes & 6 \\
\hline Chandra (2001) & Yes & Yes & Yes & No & NR & Yes & Yes & Yes & 6 \\
\hline Chen (2007) & Yes & Yes & Yes & Yes & No & Yes & Yes & Yes & 7 \\
\hline Cornelius (2004) & Yes & $\mathrm{NC}$ & NR & NR & NR & Yes & Yes & Yes & 4 \\
\hline Dahl (2007) & Yes & Yes & NR & NR & NR & Yes & Yes & Yes & 5 \\
\hline Das (2006) & Yes & Yes & Yes & Yes & Yes & Yes & Yes & Yes & 8 \\
\hline Das (2008) & Yes & Yes & Yes & Yes & Yes & Yes & Yes & Yes & 8 \\
\hline de Jesus Llibre (2009) & Yes & Yes & Yes & No & NR & Yes & Yes & Yes & 6 \\
\hline de Silva (2003) & Yes & Yes & NR & NR & NR & Yes & Yes & Yes & 5 \\
\hline Demirovic (2003) & Yes & Yes & No & Yes & No & Yes & Yes & Yes & 6 \\
\hline Di Carlo (2002) & Yes & Yes & Yes & No & No & Yes & Yes & Yes & 6 \\
\hline Ebly (1994) & Yes & Yes & Yes & NR & NR & Yes & Yes & Yes & 6 \\
\hline Edland (2002) & Yes & Yes & Yes & Yes & Yes & Yes & Yes & Yes & 8 \\
\hline Evans (2003) & Yes & Yes & NR & NR & $\mathrm{NC}$ & Yes & Yes & Yes & 5 \\
\hline Fish (2008) & Yes & Yes & Yes & Yes & $\mathrm{NC}$ & Yes & Yes & Yes & 7 \\
\hline Fitzpatrick (2004) & Yes & Yes & Yes & Yes & Yes & Yes & Yes & Yes & 8 \\
\hline Forti (2010) & Yes & Yes & Yes & Yes & No & Yes & Yes & Yes & 7 \\
\hline Fuhrer (2003) & Yes & Yes & Yes & No & Yes & Yes & Yes & No & 6 \\
\hline
\end{tabular}


Table 4. (Continued)

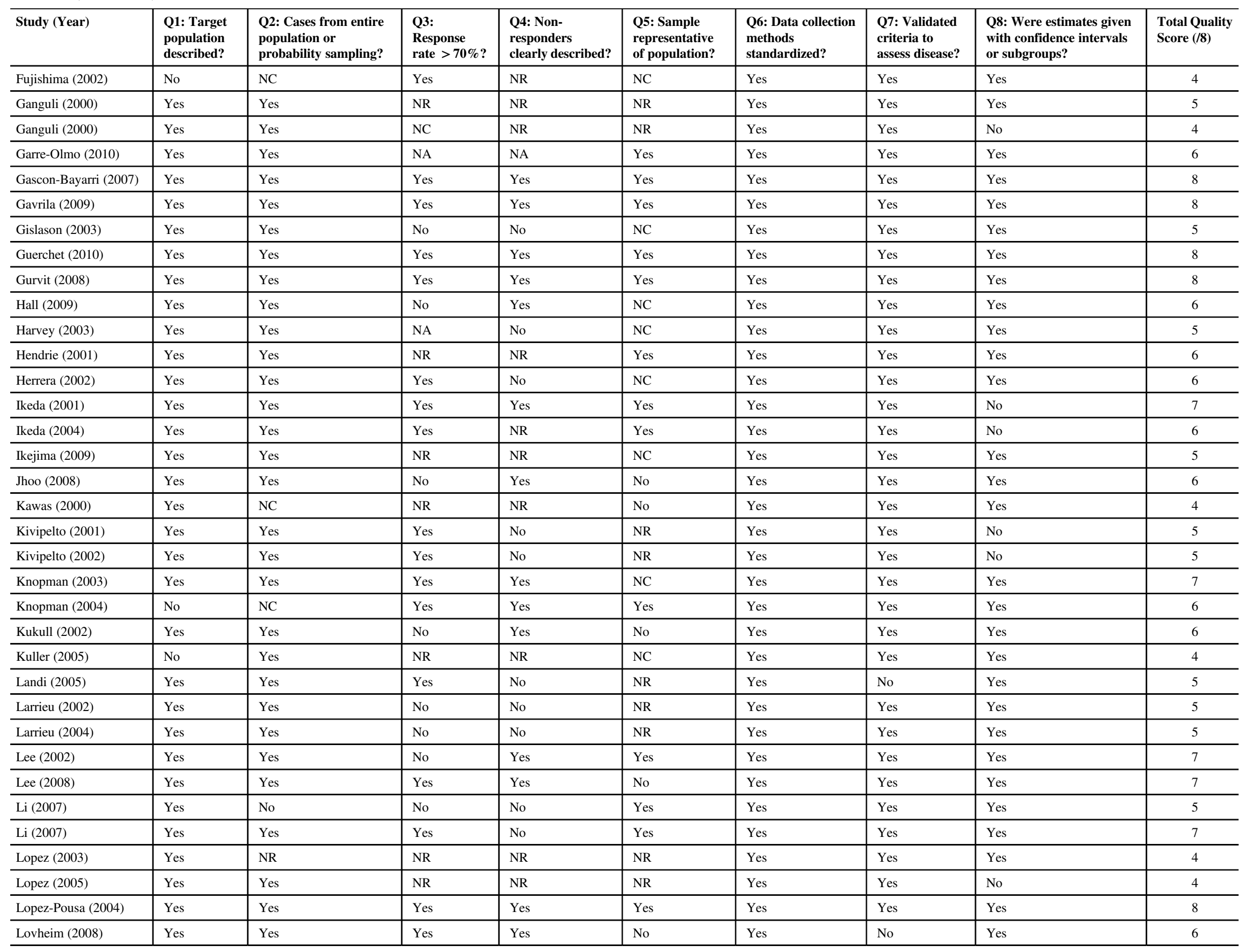




\begin{tabular}{|c|c|c|c|c|c|c|c|c|c|c|}
\hline & Magaziner (2000) & Yes & Yes & Yes & NR & Yes & Yes & Yes & Yes & 7 \\
\hline & Maneno (2006) & Yes & No & Yes & Yes & Yes & $\mathrm{NC}$ & No & No & 4 \\
\hline & Manton (2005) & Yes & Yes & NA & NR & NA & NR & No & Yes & 3 \\
\hline w & Mathuranath (2010) & Yes & Yes & NA & NA & $\mathrm{NC}$ & Yes & Yes & Yes & 5 \\
\hline & Matsui (2009) & Yes & Yes & Yes & No & $\mathrm{NC}$ & Yes & Yes & No & 5 \\
\hline 2 & McDowell (2007) & Yes & Yes & NR & NR & NR & Yes & Yes & No & 4 \\
\hline$\vec{b}$ & Meguro (2002) & Yes & Yes & No & No & NR & NR & Yes & Yes & 4 \\
\hline 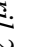 & Meguro (2007) & Yes & Yes & No & Yes & No & Yes & Yes & Yes & 6 \\
\hline 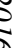 & Mercy (2008) & Yes & Yes & Yes & Yes & Yes & Yes & Yes & Yes & 8 \\
\hline & Miech (2002) & Yes & Yes & Yes & Yes & Yes & Yes & Yes & Yes & 8 \\
\hline & Molero (2007) & Yes & Yes & No & Yes & No & Yes & Yes & Yes & 6 \\
\hline & Morris (2002) & Yes & Yes & Yes & No & NR & Yes & Yes & No & 5 \\
\hline & Nitrini (2004) & Yes & Yes & NR & Yes & Yes & Yes & Yes & Yes & 7 \\
\hline & Nunes (2010) & Yes & Yes & No & No & NR & Yes & Yes & Yes & 5 \\
\hline & Perez (2010) & Yes & Yes & No & No & NR & Yes & Yes & Yes & 5 \\
\hline & Perkins (2002) & Yes & Yes & NR & NR & Yes & Yes & Yes & Yes & 6 \\
\hline & Phung (2010) & Yes & Yes & Yes & Yes & Yes & Yes & Yes & Yes & 8 \\
\hline & Piguet (2003) & Yes & Yes & Yes & No & $\mathrm{NC}$ & Yes & Yes & No & 5 \\
\hline & Plassman (2007) & Yes & Yes & No & Yes & Yes & Yes & Yes & Yes & 7 \\
\hline & Polvikoski (2001) & Yes & Yes & Yes & No & Yes & Yes & Yes & Yes & 7 \\
\hline & Polvikoski (2006) & Yes & Yes & Yes & No & Yes & Yes & Yes & No & 6 \\
\hline & Rahkonen (2003) & Yes & Yes & Yes & Yes & No & Yes & Yes & Yes & 7 \\
\hline & Ravaglia (2005) & Yes & Yes & Yes & Yes & No & Yes & Yes & Yes & 7 \\
\hline & Ravaglia (2005) & Yes & Yes & Yes & Yes & No & Yes & Yes & Yes & 7 \\
\hline & Ravalglia (2008) & Yes & Yes & Yes & Yes & No & Yes & Yes & Yes & 7 \\
\hline & Rockwood (2000) & No & Yes & No & No & $\mathrm{NR}$ & Yes & Yes & Yes & 4 \\
\hline & Rosenblatt (2004) & Yes & Yes & Yes & No & NR & Yes & Yes & No & 5 \\
\hline & Rovio (2005) & Yes & Yes & Yes & Yes & No & Yes & Yes & No & 6 \\
\hline & Ruitenberg (2001) & Yes & Yes & Yes & No & $\mathrm{NC}$ & Yes & Yes & Yes & 6 \\
\hline & Sahadevan (2008) & Yes & Yes & No & Yes & No & Yes & Yes & Yes & 6 \\
\hline & Scazufca (2008) & Yes & Yes & Yes & Yes & Yes & Yes & Yes & Yes & 8 \\
\hline & Sekita (2010) & Yes & Yes & $\mathrm{NC}$ & No & $\mathrm{NC}$ & Yes & Yes & Yes & 5 \\
\hline & Seshadri (2002) & Yes & Yes & Yes & No & $\mathrm{NC}$ & Yes & Yes & No & 5 \\
\hline & Shaji (2005) & Yes & Yes & Yes & Yes & Yes & Yes & Yes & Yes & 8 \\
\hline & Spada (2009) & Yes & Yes & Yes & No & $\mathrm{NR}$ & Yes & Yes & No & 5 \\
\hline & Stevens (2002) & Yes & Yes & Yes & Yes & NR & Yes & Yes & Yes & 7 \\
\hline & Suh (2002) & Yes & Yes & Yes & Yes & Yes & Yes & Yes & Yes & 8 \\
\hline & Tang (2001) & Yes & Yes & No & Yes & Yes & Yes & Yes & Yes & 7 \\
\hline 2 & Tyas (2006) & No & Yes & No & Yes & $\mathrm{NC}$ & Yes & Yes & Yes & 5 \\
\hline
\end{tabular}




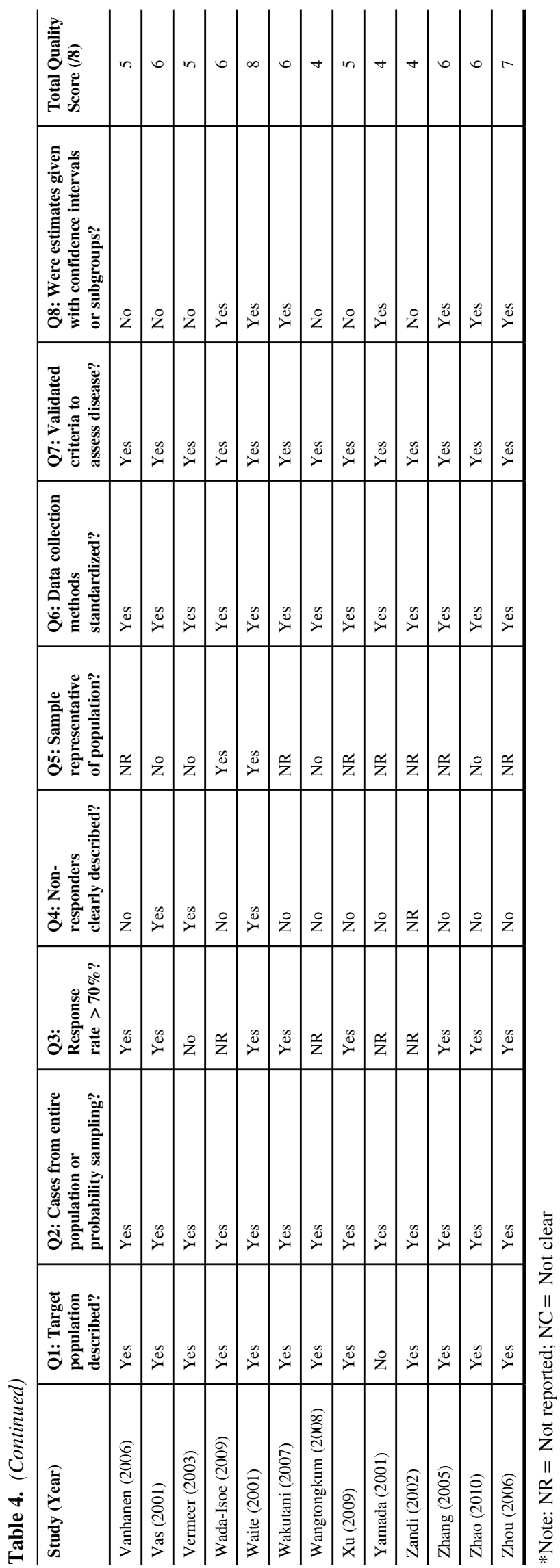

rates are falling in high-rate areas (often high-income countries) and might be rising in low- and middle-income countries, where premature mortality is decreasing. ${ }^{166,167}$ This underscores the need for future studies on the epidemiology of this important condition.

\section{Disclosures}

Kirsten Fiest, Jodie Roberts, Colleen Maxwell, Eric Smith, Alexandra Frolkis, Adrienne Cohen, Andrew Kirk, Dawn Pearson, Tamara Pringsheim, and Andres Venegas-Torres have nothing to disclose.

David Hogan holds the Brenda Strafford Foundation Chair in Geriatric Medicine, though receives no salary support from this.

Nathalie Jetté has the following disclosures: Public Health Agency of Canada, Principal Investigator, research support; Canada Research Chair in Neurological Health Services Research, Researcher, research support; Alberta Innovates Health Solutions Population Health Investigator, Researcher, research support.

\section{ACKNOWLEDGMENTS}

We thank Ms. Diane Lorenzetti, librarian at the University of Calgary, who guided the development of the search strategy for this systematic review. Our study is part of the National Population Health Study of Neurological Conditions. We acknowledge the membership of the Neurological Health Charities Canada and the Public Health Agency of Canada for their contribution to the success of this initiative. Funding for the study was provided by the Public Health Agency of Canada. The opinions expressed in this publication are those of the authors/researchers and do not necessarily reflect the official views of the Public Health Agency of Canada.

\section{Statement of Authorship}

KMF, JIR, CJM, DBH, TP and NJ contributed to study conception and design. KMF, JIR, CJM, DBH, EES, AC, AK, DP, $\mathrm{AV}-\mathrm{T}$ and NJ contributed to the acquisition of data. KMF and AF conducted the data analysis. KMF, JIR, CJM, DBH, EES and NJ participated in the interpretation of study data. All authors participated in critically revising the manuscript for important intellectual content and gave final approval for the submission of this manuscript and any further submissions of this work.

\section{SUPPLEMENTARY MATERIAL}

To view supplementary material for this article, please visit http://dx.doi.org/10.1017/cjn.2016.36

\section{REFERENCES}

1. Demakis GJ. Disability in Alzheimer's disease: causes, consequences, and economic considerations. J Health Hum Serv Adm. 2007;30:292-305.

2. Gillespie P, O'Shea E, Cullinan J, Lacey L, Gallagher D, $\mathrm{Ni}$ Mhaolain A, et al. The effects of dependence and function on costs of care for Alzheimer's disease and mild cognitive impairment in Ireland. Int J Geriatr Psychiatry. 2013;28:256-64.

3. World Health Organization. The 10 Leading Causes of Death by Broad Income Group. Geneva: World Health Organization; 2008.

4. Brookmeyer R, Evans DA, Hebert L, Langa KM, Heeringa SG, Plassman BL, et al. National estimates of the prevalence of Alzheimer's disease in the United States. Alzheimers Dement. 2011;7:61-73. 
5. Alzheimer A. Über einen eigenartigen schweren Erkrankungsproze $\beta$ der Hirnrincle. Neurol Zentral. 1906;25:1134.

6. Doody RS, Stevens JC, Beck C, Dubinsky RM, Kaye JA, Gwyther L, et al. Practice parameter: management of dementia (an evidencebased review). Report of the Quality Standards Subcommittee of the American Academy of Neurology. Neurology. 2001;56: $1154-1166$

7. Morris JC. Dementia update 2005. Alzheimer Dis Assoc Disord. 2005;19:100-17.

8. World Health Organization. Dementia: A Public Health Priority. Geneva: World Health Organization; 2012.

9. Alzheimer's Disease International. World Alzheimer Report 2015: The Global Impact of Dementia. An Analysis of Prevalence, Incidence, Cost and Trends. London: Alzheimer's Disease International; 2015.

10. Ziegler-Graham K, Brookmeyer R, Johnson E, Arrighi HM. Worldwide variation in the doubling time of Alzheimer's disease incidence rates. Alzheimers Dement. 2008;4:316-23.

11. Kukull WA, Higdon R, Bowen JD, McCormick WC, Teri L, Schellenberg GD, et al. Dementia and Alzheimer disease incidence: a prospective cohort study. Arch Neurol. 2002;59:1737-46.

12. Jorm AF, Jolley D. The incidence of dementia: a meta-analysis. Neurology. 1998;51:728-33.

13. Jorm AF, Korten AE, Henderson AS. The prevalence of dementia: a quantitative integration of the literature. Acta Psychiatr Scand. 1987;76:465-79.

14. Rockwood K, Stadnyk K. The prevalence of dementia in the elderly: a review. Can J Psychiatry. 1994;39:253-7.

15. Fratiglioni L, De Ronchi D, Aguero-Torres H. Worldwide prevalence and incidence of dementia. Drugs Aging. 1999;15: 365-375.

16. Clarfield AM. The decreasing prevalence of reversible dementias: an updated meta-analysis. Arch Intern Med. 2003;163:2219-29.

17. Suh GH, Shah A. A review of the epidemiological transition in dementia: cross-national comparisons of the indices related to Alzheimer's disease and vascular dementia. Acta Psychiatr Scand. 2001;104:4-11

18. Caesar-Chavannes C, MacDonald S. Cross-Canada Forum: National Population Health Study of Neurological Conditions in Canada. Chronic Dis Inj Can. 2013;33:188-91.

19. Moher D, Liberati A, Tetzlaff J, Altman DG, PRISMA Group. Preferred reporting items for systematic reviews and meta-analyses: the PRISMA statement. PLoS Med. 2009;6(7): e1000097.

20. EndNote X5. . New York: Thompson Reuters, 2011. Available from http://endnote.com/downloads/available-updates.

21. Loney PL, Chambers LW, Bennett KJ, Roberts JG, Stratford PW. Critical appraisal of the health research literature: prevalence or incidence of a health problem. Chronic Dis Inj Can. 1998;19:170-6.

22. Boyle MH.. Guidelines for evaluating prevalence studies. Evid Based Ment Health. 1998;1:37-9.

23. Begg C, Mazumdar M.. Operating characteristics of a rank correlation test for publication bias. Biometrics. 1994;50:1088-101.

24. Egger M, Smith G. Bias in meta-analysis detected by simple, graphical test. Br Med J. 1997;315:629-34.

25. R: A Language and Environment for Statistical Computing [computer program]. Vienna, Austria: R Foundation for Statistical Computing; 2011.

26. Schwarzer G. meta: Meta-Analysis with R. R package version 16-1; 2010.

27. Viechtbauer W. Conducting meta-analyses in $\mathrm{R}$ with the metafor package. J Stat Softw. 2010;36:1-48.

28. Canadian study of health and aging: study methods and prevalence of dementia. CMAJ. 1994;150:899-913.

29. Anttila T, Helkala EL, Viitanen M, Kareholt I, Fratiglioni L, Winblad $\mathrm{B}$, et al. Alcohol drinking in middle age and subsequent risk of mild cognitive impairment and dementia in old age: a prospective population based study. BMJ. 2004;329:539.

30. Benedetti MD, Salviati A, Filipponi S, Manfredi M, De Togni L, Gomez Lira M, et al. Prevalence of dementia and apolipoprotein E genotype distribution in the elderly of Buttapietra, Verona Province, Italy. Neuroepidemiology. 2002;21:74-80.
31. Bermejo-Pareja F, Benito-Leon J, Vega S, Olazaran J, de Toledo M, Diaz-Guzman J, et al. Consistency of clinical diagnosis of dementia in NEDICES: A population-based longitudinal study in Spain. J Geriatr Psychiatry Neurol. 2009;22:246-55.

32. Borroni B, Alberici A, Grassi M, Rozzini L, Turla M, Zanetti O, et al. Prevalence and demographic features of early-onset neurodegenerative dementia in Brescia County, Italy. Alzheimer Dis Assoc Disord. 2011;25:341-4.

33. Bottino CMC, Azevedo D Jr, Tatsch M, Hototian SR, Moscoso MA, Folquitto J, et al. Estimate of dementia prevalence in a community sample from São Paulo, Brazil. Dement Geriatr Cogn Disord. 2008;26:291-9.

34. Bowirrat A, Treves TA, Friedland RP, Korczyn AD. Prevalence of Alzheimer's type dementia in an elderly Arab population. Eur J Neurol. 2001;8:119-23.

35. Camicioli R, Willert P, Lear J, Grossmann S, Kaye J, Butterfield P. Dementia in rural primary care practices in Lake County, Oregon. J Geriatr Psychiatry Neurol. 2000;13:87-92.

36. Chen TF, Chiu MJ, Tang LY, Chiu YH, Chang SF, Su CL, et al. Institution type-dependent high prevalence of dementia in longterm care units. Neuroepidemiology. 2007;28:142-9.

37. Dahl A, Berg S, Nilsson SE. Identification of dementia in epidemiological research: a study on the usefulness of various data sources. Aging Clin Exp Res. 2007;19:381-9.

38. de Jesus Llibre J, Fernandez Y, Marcheco B, Contreras N, Lopez AM, Otero M, et al. Prevalence of dementia and Alzheimer's disease in a Havana municipality: a community-based study among elderly residents. MEDICC Rev. 2009;11:29-35.

39. de Silva HA, Gunatilake SB, Smith AD. Prevalence of dementia in a semi-urban population in Sri Lanka: report from a regional survey. Int J Geriatr Psychiatry. 2003;18:711-5.

40. Fish M, Bayer AJ, Gallacher JEJ, Bell T, Pickering J, Pedro S, et al. Prevalence and pattern of cognitive impairment in a community cohort of men in South Wales: methodology and findings from the Caerphilly Prospective Study. Neuroepidemiology. 2008;30:25-33.

41. Fujishima M, Kiyohara Y.. Incidence and risk factors of dementia in a defined elderly Japanese population: the Hisayama study. Ann N Y Acad Sci. 2002;977:1-8.

42. Gurvit H, Emre M, Tinaz S, Bilgic B, Hanagasi H, Sahin H, et al. The prevalence of dementia in an urban Turkish population. Am J Alzheimers Dis Other Demen. 2008;23:67-76.

43. Hall KS, Gao S, Baiyewu O, Lane KA, Gureke O, Shen J, et al. Prevalence rates for dementia and Alzheimer's disease in African Americans: 1992 versus 2001. Alzheimer's Dement. 2009;5: 227-233.

44. Harvey RJ, Skelton-Robinson M, Rossor MN. The prevalence and causes of dementia in people under the age of 65 years. J Neurol Neurosurg Psychiatry. 2003;74:1206-9.

45. Herrera E Jr, Caramelli P, Silveira ASB, Nitrini R. Epidemiologic survey of dementia in a community-dwelling Brazilian population. Alzheimer Dis Assoc Disord. 2002;16:103-8.

46. Ikeda M, Hokoishi K, Maki N, Nebu A, Tachibana N, Komori K, et al. Increased prevalence of vascular dementia in Japan: a communitybased epidemiological study. Neurology. 2001;57:839-44.

47. Ikejima C, Yasuno F, Mizukami K, Sasaki M, Tanimukai S, Asada T. Prevalence and causes of early-onset dementia in Japan: a population-based study. Stroke. 2009;40:2709-14

48. Kivipelto M, Helkala EL, Laakso M, Hanninen T, Hallikainen M, Alhainen K, et al. Apolipoprotein E e4 allele, elevated midlife total cholesterol level, and high midlife systolic blood pressure are independent risk factors for late-life Alzheimer disease. Ann Intern Med. 2002;137:149-55.

49. Kivipelto M, Helkala EL, Laakso M, Hanninen T, Hallikainen M, Alhainen K, et al.. Midlife vascular risk factors and Alzheimer's disease in later life: longitudinal, population based study. BMJ. 2001;322:1447-51.

50. Mathuranath PS, Cherian PJ, Mathew R, Kumar S, George A, Alexander A, et al. Dementia in Kerala, South India: prevalence and influence of age, education and gender. Int J Geriatr Psychiatry. 2010;25:290-7.

51. Meguro K, Ishii H, Yamaguchi S, Ishizaki J, Shimada M, Sato M, et al. Prevalence of dementia and dementing diseases in Japan: the Tajiri Project. Arch Neurol. 2002;59:1109-14. 
52. Nunes B, Silva RD, Cruz VT, Roriz JM, Pais J, Silva MC. Prevalence and pattern of cognitive impairment in rural and urban populations from Northern Portugal. BMC Neurol. 2010;10:42.

53. Plassman BL, Langa KM, Fisher GG, Fisher GG, Heeringa SG, Weir DR, et al. Prevalence of dementia in the United States: the aging, demographics, and memory study. Neuroepidemiology. 2007;29:125-32.

54. Polvikoski T, Sulkava R, Myllykangas L, Notkola IL, Niinisto L, Verkkoniemi A, et al. Prevalence of Alzheimer's disease in very elderly people: a prospective neuropathological study. Neurology. 2001;56:1690-6.

55. Rahkonen T, Eloniemi-Sulkava U, Rissanen S, Vatanen A, Viramo P, Sulkava R. Dementia with Lewy bodies according to the consensus criteria in a general population aged 75 years or older. J Neurol Neurosurg Psychiatry. 2003;74:720-4.

56. Rosenblatt A, Samus QM, Steele CD, Baker AS, Harper MG, Brandt J, et al. The Maryland Assisted Living Study: prevalence, recognition, and treatment of dementia and other psychiatric disorders in the assisted-living population of central Maryland. J Am Geriatr Soc. 2004;52:1618-25.

57. Rovio S, Kareholt I, Helkala EL, Viitanen M, Winblad B, Tuomilehto $\mathrm{J}$, et al. Leisure-time physical activity and the risk of dementia and Alzheimer's disease. Lancet Neurol. 2005; 4:705-11.

58. Sahadevan S, Saw SM, Gao W, Tan LC, Chin JJ, Hong CY, et al. Ethnic differences in Singapore's dementia prevalence: the stroke, Parkinson's disease, epilepsy, and dementia in Singapore study. J Am Geriatr Soc. 2008;56:2061-8.

59. Sekita A, Ninomiya T, Tanizaki Y, Doi Y, Hata J, Yonemoto K, et al. Trends in prevalence of Alzheimer's disease and vascular dementia in a Japanese community: the Hisayama Study. Acta Psychiatr Scand. 2010;122:319-25.

60. Shaji S, Bose S, Verghese A. Prevalence of dementia in an urban population in Kerala, India. Br J Psychiatry. 2005;187:90.

61. Spada RS, Stella G, Calabrese S, Bosco P, Anello G, Gueant-Rodriguez RM, et al. Prevalence of dementia in mountainous village of Sicily. J Neurol Sci. 2009;283:62-5.

62. Stevens T, Livingston G, Kitchen G, Manela M, Walker Z, Katona C. Islington study of dementia subtypes in the community. $\mathrm{Br} \mathrm{J}$ Psychiatry. 2002;180:270-6.

63. Suh GH, Kim JK, Cho MJ. Community study of dementia in the older Korean rural population. Aust N Z J Psychiatry. 2003;37:606-12.

64. Vas CJ, Pinto C, Panikker D, Noronha S, Deshpande N, Kulkarni L, et al. Prevalence of dementia in an urban Indian population. Int Psychogeriatr. 2001;13:439-50.

65. Wada-Isoe K, Uemura Y, Suto Y, Doi K, Imamura K, Hayashi A, et al. Prevalence of dementia in the rural island town of Ama-cho, Japan. Neuroepidemiology. 2009;32:101-6.

66. Wakutani Y, Kusumi M, Wada K, Kawashima M, Ishizaki K, Mori M, et al. Longitudinal changes in the prevalence of dementia in a Japanese rural area. Psychogeriatrics. 2007;7:150-4.

67. Yamada T, Hattori H, Miura A, Tanabe M, Yamori Y. Prevalence of Alzheimer's disease, vascular dementia and dementia with Lewy bodies in a Japanese population. Psychiatry Clin Neurosci. 2001;55:21-5.

68. Zhang ZX, Zahner GEP, Román GC, Liu J, Hong Z, Qu QM, et al. Dementia subtypes in China: prevalence in Beijing, Xian, Shanghai, and Chengdu. Arch Neurol. 2005;62:447-53.

69. Zhou DF, Wu CS, Qi H, Fan JH, Sun XD, Como P, et al. Prevalence of dementia in rural China: impact of age, gender and education. Acta Neurol Scand. 2006;114:273-80.

70. Bowirrat A, Friedland RP, Farrer L, Baldwin C, Korczyn A. Genetic and environmental risk factors for Alzheimer's disease in Israeli Arabs. J Mol Neurosci. 2002;19:239-45.

71. Bowirrat A, Friedland RP, Korczyn AD. Vascular dementia among elderly Arabs in Wadi Ara. J Neurol Sci. 2002;203-4:73-6.

72. Li S, Yan F, Li G, Zhang W, Liu J, Jie X, et al. Is the dementia rate increasing in Beijing? Prevalence and incidence of dementia 10 years later in an urban elderly population. Acta Psychiatr Scand. 2007;115:73-9.

73. Andreasen N, Blennow K, Sjodin C, Winblad B, Svardsudd K. Prevalence and incidence of clinically diagnosed memory impairments in a geographically defined general population in Sweden: The Piteå Dementia Project. Neuroepidemiology. 1999;18:144-55.

74. Arslantas D, Ozbabalik D, Metintas S, Ozkan S, Kalyoncu C, Ozdemir G, et al. Prevalence of dementia and associated risk factors in Middle Anatolia, Turkey. J Clin Neurosci. 2009;16:1455-9.

75. Banerjee TK, Mukherjee CS, Dutt A, Shekhar A, Hazra A. Cognitive dysfunction in an urban Indian population: some observations. Neuroepidemiology. 2008;31:109-14.

76. Borjesson-Hanson A, Edin E, Gislason T, Skoog I.. The prevalence of dementia in 95 year olds. Neurology. 2004;63:2436-8.

77. Das SK, Biswas A, Roy J, Bose P, Roy T, Banerjee TK, et al. Prevalence of major neurological disorders among geriatric population in the metropolitan city of Kolkata. J Assoc Physicians India. 2008;56:175-81

78. Das SK, Biswas A, Roy T, Banerjee TK, Mukherjee CS, Raut DK, et al. A random sample survey for prevalence of major neurological disorders in Kolkata. Indian J Med Res. 2006;124:163-72.

79. Demirovic J, Prineas R, Loewenstein D, Bean J, Duara R, Sevush S, et al. Prevalence of dementia in three ethnic groups: the South Florida program on aging and health. Ann Epidemiol. 2003; $13: 472-8$.

80. Ebly EM, Parhad IM, Hogan DB, Fung TS. Prevalence and types of dementia in the very old: results from the Canadian Study of Health and Aging. Neurology. 1994;44:1593-600.

81. Ganguli M, Chandra V, Kamboh MI, Johnston JM, Dodge HH, Thelma BK, et al. Apolipoprotein E polymorphism and Alzheimer disease: The Indo-US Cross-National Dementia Study. Arch Neurol. 2000;57:824-30.

82. Gascon-Bayarri J, Rene R, Del Barrio JL, De Pedro-Cuesta J, Ramon JM, Manubens JM, et al. Prevalence of dementia subtypes in El Prat de Llobregat, Catalonia, Spain: the PRATICON study. Neuroepidemiology. 2007;28:224-34.

83. Gavrila D, Antunez C, Tormo MJ, Carles R, Garcia Santos JM, Parrilla G, et al. Prevalence of dementia and cognitive impairment in Southeastern Spain: the Ariadna study. Acta Neurol Scand. 2009;120:300-7.

84. Gislason TB, Sjogren M, Larsson L, Skoog I. The prevalence of frontal variant frontotemporal dementia and the frontal lobe syndrome in a population-based sample of 85 year olds. J Neurol Neurosurg Psychiatry. 2003;74:867-71.

85. Guerchet M, M'Belesso P, Mouanga AM, Bandzouzi B, Tabo A, Houinato DS, et al. Prevalence of dementia in elderly living in two cities of Central Africa: the EDAC survey. Dement Geriatr Cogn Disord. 2010;30:261-8.

86. Ikeda M, Fukuhara R, Shigenobu K, Hokoishi K, Maki N, Nebu A, et al. Dementia-associated mental and behavioural disturbances in elderly people in the community: findings from the first Nakayama study. J Neurol Neurosurg Psychiatry. 2004;75:146-8.

87. Jhoo JH, Kim KW, Huh Y, Lee SB, Park JH, Lee JJ, et al. Prevalence of dementia and its subtypes in an elderly urban Korean population: results from the Korean Longitudinal Study on Health and Aging (KLoSHA). Dement Geriatr Cogn Disord. 2008; 26:270-6.

88. Landi F, Russo A, Cesari M, Barillaro C, Onder G, Zamboni V, et al. The ilSIRENTE study: a prospective cohort study on persons aged 80 years and older living in a mountain community of Central Italy. Aging Clin Exp Res. 2005;17:486-93.

89. Lee DY, Lee JH, Ju YS, Lee KU, Kim KW, Jhoo JH, et al. The prevalence of dementia in older people in an urban population of Korea: the Seoul study. J Am Geriatr Soc. 2002;50:1233-9.

90. Li G, Rhew IC, Shofer JB, Rhew IC, Kukull WA, Peskind ER, et al. Age-varying association between blood pressure and risk of dementia in those aged 65 and older: a community-based prospective cohort study. J Am Geriatr Soc. 2007;55:1161-7.

91. Lopez OL, Kuller LH, Fitzpatrick A, Ives D, Becker JT, Beauchamp $\mathrm{N}$. Evaluation of dementia in the cardiovascular health cognition study. Neuroepidemiology. 2003;22:1-12.

92. Lovheim H, Karlsson S, Gustafson Y. The use of central nervous system drugs and analgesics among very old people with and without dementia. Pharmacoepidemiol Drug Saf. 2008; 17:912-8. 
93. Magaziner J, German P, Zimmerman SI, Hebel JR, Burton L, Gruber-Baldini AL, et al. The prevalence of dementia in a statewide sample of new nursing home admissions aged 65 and older: diagnosis by expert panel. Epidemiology of Dementia in Nursing Homes Research Group. Gerontologist. 2000;40:663-72.

94. Maneno MK, Lee E, Wutoh AK, Zuckerman IH, Jackson P, Lombardo FA, et al. National patterns of dementia treatment among elderly ambulatory patients. J Natl Med Assoc. 2006;98:430-5.

95. Manton KC, Gu XL, Ukraintseva SV. Declining prevalence of dementia in the U.S. elderly population. Adv Gerontol. 2005; 16:30-7.

96. Molero AE, Pino-Ramirez G, Maestre GE. High prevalence of dementia in a Caribbean population. Neuroepidemiology. 2007;29:107-12.

97. Perkins AJ, Hui SL, Ogunniyi A, Gureje O, Baiyewu O, Unverzaqt FW, et al. Risk of mortality for dementia in a developing country: the Yoruba in Nigeria. Int J Geriatr Psychiatry. 2002;17:566-73.

98. Rockwood K, Wentzel C, Hachinski V, Hogan DB, MacKnight C, McDowell I. Prevalence and outcomes of vascular cognitive impairment: Vascular Cognitive Impairment Investigators of the Canadian Study of Health and Aging. Neurology. 2000;54:447-51.

99. Scazufca M, Menezes PR, Vallada HP, Crepaldi AL, Pastor-Valero M, Coutinho LM, et al. High prevalence of dementia among older adults from poor socioeconomic backgrounds in São Paulo, Brazil. Int Psychogeriatr. 2008;20:394-405.

100. Vanhanen M, Koivisto K, Moilanen L, Helkala EL, Hanninen T, Soininen $\mathrm{H}$, et al. Association of metabolic syndrome with Alzheimer disease: a population-based study. Neurology. 2006;67:843-7.

101. Wangtongkum S, Sucharitkul P, Silprasert N, Inthrachak R. Prevalence of dementia among population age over 45 years in Chiang Mai, Thailand. J Med Assoc Thai. 2008;91:1685-90.

102. Xu W, Qiu C, Gatz M, Pedersen NL, Johansson B, Fratiglioni L. Mid- and late-life diabetes in relation to the risk of dementia: a population-based twin study. Diabetes. 2009;58:71-7.

103. Zhao Q, Zhou B, Ding D, Guo Q, Hong Z. Prevalence, mortality, and predictive factors on survival of dementia in Shanghai, China. Alzheimer Dis Assoc Disord. 2010;24:151-8.

104. Cornelius C, Fastbom J, Winblad B, Viitanen M. Aspirin, NSAIDs, risk of dementia, and influence of the apolipoprotein E epsilon 4 allele in an elderly population. Neuroepidemiology. 2004;23: 135-143.

105. Evans DA, Bennett DA, Wilson RS, Bienias JL, Morris MC, Scherr PA, et al. Incidence of Alzheimer disease in a biracial urban community: relation to apolipoprotein E allele status. Arch Neurol. 2003;60:185-9.

106. Forti P, Pisacane N, Rietti E, Lucicesare A, Olivelli V, Mariani E, et al. Metabolic syndrome and risk of dementia in older adults. J Am Geriatr Soc. 2010;58:487-92.

107. Ganguli M, Dodge HH, Chen P, Belle S, DeKosky ST. Ten-year incidence of dementia in a rural elderly US community population: the MoVIES Project. Neurology. 2000;54:1109-16.

108. Hendrie HC, Ogunniyi A, Hall KS, Baiyewu O, Unverzagt FW, Gureje $\mathrm{O}$, et al. Incidence of dementia and Alzheimer disease in two communities: Yoruba residing in Ibadan, Nigeria, and African Americans residing in Indianapolis, Indiana. JAMA. 2001;285:739-47.

109. Kawas C, Gray S, Brookmeyer R, Fozard J, Zonderman A. Age-specific incidence rates of Alzheimer's disease: the Baltimore Longitudinal Study of Aging. Neurology. 2000;54:2072-7.

110. Knopman DS, Rocca WA, Cha RH, Edland SD, Kokmen E. Survival study of vascular dementia in Rochester, Minnesota. Arch Neurol. 2003;60:85-90.

111. Kuller LH, Lopez OL, Jagust WJ, Becker JT, DeKosky ST, Lyketsos $\mathrm{C}$, et al. Determinants of vascular dementia in the Cardiovascular Health Cognition Study. Neurology. 2005;64:1548-1552.

112. Lopez OL, Kuller LH, Becker JT, Jaqust WJ, DeKosky ST, Fitzpatrick A, et al. Classification of vascular dementia in the Cardiovascular Health Study Cognition Study. Neurology. 2005;64:1539-47.
113. Miech RA, Breitner JCS, Zandi PP, Khachaturian AS, Anthony JC, Mayer L. Incidence of AD may decline in the early 90 s for men, later for women: The Cache County Study. Neurology. 2002;58:209-18.

114. Morris MC, Evans DA, Bienias JL, Tangney CC, Bennett DA, Aggarwal N, et al. Dietary intake of antioxidant nutrients and the risk of incident Alzheimer disease in a biracial community study. JAMA. 2002;287:3230-7.

115. Piguet O, Grayson DA, Creasey H, Bennett HP, Brooks WS, Waite $\mathrm{LM}$, et al. Vascular risk factors, cognition and dementia incidence over 6 years in the Sydney Older Persons Study. Neuroepidemiology. 2003;22:165-71.

116. Seshadri S, Beiser A, Selhub J, Jacques PF, Rosenberg IH, D'Agostino RB, et al. Plasma homocysteine as a risk factor for dementia and Alzheimer's Disease. N Engl J Med. 2002;346: 476-483.

117. Zandi PP, Carlson MC, Plassman BL, Welsh-Bohmer KA, Mayer LS, Steffens DC, et al. Hormone replacement therapy and incidence of Alzheimer disease in older women: The Cache County Study. JAMA. 2002;288:2123-9.

118. Bermejo-Pareja F, Benito-León J, Vega S, Medrano MJ, Román GC, Neurological Disorders in Central Spain (NEDICES) Study Group. Incidence and subtypes of dementia in three elderly populations of central Spain. J Neurol Sci. 2008;264:63-72.

119. Chandra V, Pandav R, Dodge HH, Johnston JM, Belle SH, DeKosky ST, et al. Incidence of Alzheimer's disease in a rural community in India: the Indo-US study. Neurology. 2001;57: 985-989.

120. Di Carlo A, Baldereschi M, Amaducci L, Lepore V, Bracco L, Maggi S, et al. Incidence of dementia, Alzheimer's disease, and vascular dementia in Italy: The ILSA Study. J Am Geriatr Soc. 2002;50:41-8.

121. Edland SD, Rocca WA, Petersen RC, Cha RH, Kokmen E. Dementia and Alzheimer disease incidence rates do not vary by sex in Rochester, Minnesota. Arch Neurol. 2002;59:1589-93.

122. Fitzpatrick AL, Kuller LH, Ives DG, Lopez OL, Jagust W, Breitner $\mathrm{JC}$, et al. Incidence and prevalence of dementia in the Cardiovascular Health Study. J Am Geriatr Soc. 2004;52:195-204.

123. Fuhrer R, Dufouil C, Dartigues JF, PAQUID Study. Exploring sex differences in the relationship between depressive symptoms and dementia incidence: prospective results from the PAQUID Study. J Am Geriatr Soc. 2003;51:1055-63.

124. Garre-Olmo J, Genis Batlle D, del Mar Fernandez M, Marquez Daniel F, de Eugenio Huelamo R, Casadevall T, et al. Incidence and subtypes of early-onset dementia in a geographically defined general population. Neurology. 2010;75:1249-55.

125. Knopman DS, Petersen RC, Edland SD, Cha RH, Rocca WA. The incidence of frontotemporal lobar degeneration in Rochester, Minnesota, 1990 through 1994. Neurology. 2004;62:506-8.

126. Kukull WA, Higdon R, Bowen JD, McCormick WC, Teri L, Schellenberg GD, et al. Dementia and Alzheimer disease incidence: a prospective cohort study. Arch Neurol. 2002;59: 1737-46.

127. Larrieu S, Letenneur L, Helmer C, Dartigues JF, Barberger-Gateau P. Nutritional factors and risk of incident dementia in the PAQUID longitudinal cohort. J Nutr Health Aging. 2004;8:150-4.

128. Larrieu S, Letenneur L, Orgogozo JM, Fabrigoule C, Amieva H, Le Carret $\mathrm{N}$, et al. Incidence and outcome of mild cognitive impairment in a population-based prospective cohort. Neurology. 2002;59:1594-9.

129. Lee JY, Chang SM, Jang HS, Chang JS, Suh GH, Jung HY, et al. Illiteracy and the incidence of Alzheimer's disease in the Yonchon County survey, Korea. Int Psychogeriatr. 2008;20:976-85.

130. Lopez-Pousa S, Vilalta-Franch J, Llinas-Regla J, Garre-Olmo J, Román GC. Incidence of dementia in a rural community in Spain: the Girona cohort study. Neuroepidemiology. 2004;23:170-7.

131. Matsui Y, Tanizaki Y, Arima H, Yonemoto K, Doi Y, Ninomiya T, et al. Incidence and survival of dementia in a general population of Japanese elderly: the Hisayama study. J Neurol Neurosurg Psychiatry. 2009;80:366-70.

132. McDowell I, Xi G, Lindsay J, Tierney M. Mapping the connections between education and dementia. J Clin Exp Neuropsychol. 2007;29:127-41. 
133. Meguro K, Ishii $\mathrm{H}$, Kasuya $\mathrm{M}$, Akanuma K, Meguro M, Kasai M, et al. Incidence of dementia and associated risk factors in Japan: The Osaki-Tajiri Project. J Neurol Sci. 2007;260:175-82.

134. Mercy L, Hodges JR, Dawson K, Barker RA, Brayne C. Incidence of early-onset dementias in Cambridgeshire, United Kingdom. Neurology. 2008;71:1496-9.

135. Nitrini R, Caramelli P, Herrera E Jr, Bahia VS, Caixeta LF, Radanovic $\mathrm{M}$, et al. Incidence of dementia in a communitydwelling Brazilian population. Alzheimer Dis Assoc Disord. 2004;18:241-6.

136. Perez F, Helmer C, Dartigues JF, Auriacombe S, Tison F. A 15-year population-based cohort study of the incidence of Parkinson's disease and dementia with Lewy bodies in an elderly French cohort. J Neurol Neurosurg Psychiatry. 2010;81:742-6.

137. Phung TKT, Waltoft BL, Kessing LV, Mortensen PB, Waldemar G. Time trend in diagnosing dementia in secondary care. Dement Geriatr Cogn Disord. 2010;29:146-53.

138. Polvikoski T, Sulkava R, Rastas S, Sutela A, Niinisto L, Notkola IL, et al. Incidence of dementia in very elderly individuals: a clinical, neuropathological and molecular genetic study. Neuroepidemiology. 2006;26:76-82.

139. Ravaglia G, Forti P, Lucicesare A, Piscane N, Rietti E, Biachin M, et al. Physical activity and dementia risk in the elderly: findings from a prospective Italian study. Neurology. 2008;70:1786-94.

140. Ravaglia G, Forti P, Maioli F, Martelli M, Servadei L, Brunetti N, et al. Incidence and etiology of dementia in a large elderly Italian population. Neurology. 2005;64:1525-30.

141. Ravaglia G, Forti P, Maioli F, Montesi F, Rietti E, Pisacane N, et al. Risk factors for dementia: data from the Conselice study of brain aging. Arch Gerontol Geriatr. 2007;44(Suppl 1):311-20.

142. Ruitenberg A, Ott A, van Swieten JC, Hofman A, Breteler MM. Incidence of dementia: does gender make a difference? Neurobiol Aging. 2001;22:575-80.

143. Tang MX, Cross P, Andrews H, Jacobs DM, Small S, Bell K, et al. Incidence of $\mathrm{AD}$ in African Americans, Caribbean Hispanics, and Caucasians in northern Manhattan. Neurology. 2001;56:49-56.

144. Tyas SL, Tate RB, Wooldrage K, Manfreda J, Strain LA. Estimating the incidence of dementia: the impact of adjusting for subject attrition using health care utilization data. Ann Epidemiol. 2006; $16: 477-84$

145. Vermeer SE, Prins ND, den Heijer T, Hofman A, Koudstaal PJ, Breteler MM. Silent brain infarcts and the risk of dementia and cognitive decline. N Engl J Med. 2003;348:1215-22.

146. Waite LM, Broe GA, Grayson DA, Creasey H. The incidence of dementia in an Australian community population: the Sydney Older Persons Study. Int J Geriatr Psychiatry. 2001;16:680-9.

147. American Psychiatric Association. Diagnostic and Statistical Manual of Mental Disorders-IV-Text Revision. Washington, DC: American Psychiatric Association; 1994

148. McKhann G, Drachman D, Folstein M, Katzman R, Price D, Stadlan EM. Clinical diagnosis of Alzheimer's disease: report of the NINCDS-ADRDA Work Group under the auspices of the Department of Health and Human Services Task Force on Alzheimer's Disease. Neurology. 1984;34:939-44.

149. Hendrie HC, Osuntokun BO, Hall KS, Ogunniyi AO, Hui SL, Unverzagt FW, et al. Prevalence of Alzheimer's disease and dementia in two communities: Nigerian Africans and African Americans. Am J Psychiatr. 1995; 152:1485-92.

150. Danila O, Hirdes JP, Maxwell CJ, Marrie RA, Patten S, Pringsheim T, Jetté N. Prevalence of neurological conditions across the continuum of care based on interRAI assessments. BMC Health Serv Res. 2014;14:29.
151. Hebert LE, Weuve J, Scherr PA, Evans DA. Alzheimer disease in the United States (2010-2050) estimated using the 2010 census. Neurology. 2013;80:1778-83.

152. Andersen K, Launer LJ, Dewey ME, Letenneur L, Ott A, Copeland JR, et al. Gender differences in the incidence of $\mathrm{AD}$ and vascular dementia: The EURODEM Studies. EURODEM Incidence Research Group. Neurology. 1999;53:1992-7.

153. Fratiglioni L, Launer LJ, Andersen K, Breteler MM, Copeland JR, Dartiques JF, et al. Incidence of dementia and major subtypes in Europe: a collaborative study of population-based cohorts. Neurologic Diseases in the Elderly Research Group. Neurology. 2000;54:S10-5

154. Mielke MM, Vemuri P, Rocca WA. Clinical epidemiology of Alzheimer disease: assessing sex and gender differences. Clin Epidemiol. 2014;6:37-48.

155. Chene G, Beiser A, Au R, Preis SR, Wolf PA, Dufouil C, et al. Gender and incidence of dementia in the Framingham Heart Study from mid-adult life. Alzheimers Dement. 2015;11:310-20.

156. World Health Organization. The ICD-10 Classification of Mental and Behavioural Disorders: Diagnostic Criteria for Research. Geneva: World Health Organization; 1993.

157. Erkinjuntti T, Ostbye T, Steenhuis R, Hachinski V. The effect of different diagnostic criteria on the prevalence of dementia. N Engl J Med. 1997;337:1667-74.

158. Wilson RS, Weir DR, Leurgans SE, Evans DA, Hebert LE, Langa $\mathrm{KM}$, et al. Sources of variability in estimates of the prevalence of Alzheimer's disease in the United States. Alzheimers Dement. 2011;7:74-9.

159. McKhann GM, Knopman DS, Chertkow H, Hyman BT, Jack CR Jr, Kawas CH, et al. The diagnosis of dementia due to Alzheimer's disease: recommendations from the National Institute on Aging-Alzheimer's Association workgroups on diagnostic guidelines for Alzheimer's disease. Alzheimers Dement. 2011;7:263-9.

160. Dubois B, Feldman HH, Jacova C, Hampel H, Molinuevo JL, Blennow $\mathrm{K}$, et al. Advancing research diagnostic criteria for Alzheimer's disease: the IWG-2 criteria. Lancet Neurol. 2014;13:614-29.

161. Lopez OL, McDade E, Riverol M, Becker JT. Evolution of the diagnostic criteria for degenerative and cognitive disorders. Curr Opin Neurol. 2011;24:532-41.

162. World Health Organization. Life Expectancy at Birth. Geneva: World Health Organization; 2011.

163. Pringsheim T, Jetté N, Frolkis A, Steeves TD. The prevalence of Parkinson's disease: a systematic review and meta-analysis. Mov Disord. 2014:291583-90.

164. Pringsheim T, Wiltshire K, Day L, Dykeman J, Steeves T, Jetté N. The incidence and prevalence of Huntington's disease: a systematic review and meta-analysis. Mov Disord. 2012;27: 1083-1091.

165. Norton S, Matthews FE, Barnes D, Yaffe K, Brayne C. Potential for primary prevention of Alzheimer's disease: an analysis of population-based data. Lancet Neurol. 2014;13:788-94.

166. Schrijvers E, Verhaaren B, Koudstaal P, Hofman A, Ikram MA, Breteler MM. Is dementia incidence declining? Trends in dementia incidence since 1990 in the Rotterdam Study. Neurology. 2012;78:1456-63.

167. Matthews FE, Arthur A, Barnes LE, Bond J, Jagger C, Robinson L, et al. A two-decade comparison of prevalence of dementia in individuals aged 65 years and older from three geographical areas of England: results of the Cognitive Function and Ageing Study I and II. Lancet. 2013;382:1405-12. 